\title{
Iterative learning fault-tolerant control for differential time-delay batch processes in finite frequency domains ${ }^{\frac{t^{2}}{2}}$
}

\author{
Hongfeng Tao ${ }^{\mathrm{a}}$, Wojciech Paszke ${ }^{\mathrm{b}, *}$, Eric Rogers ${ }^{\mathrm{c}}$, Huizhong Yang $^{\mathrm{a}}$, Krzysztof Gałkowski ${ }^{\mathrm{b}}$ \\ ${ }^{a}$ Key Laboratory of Advanced Process Control for Light Industry of Ministry of Education, \\ Jiangnan University, Wuxi 214122, P. R. China \\ ${ }^{b}$ Institute of Control and Computation Engineering, University of Zielona Góra, \\ ul. Szafrana 2, 65-516 Zielona Góra, Poland \\ ${ }^{c}$ Department of Electronics and Computer Science, University of Southampton, \\ Southampton SO17 1BJ, United Kingdom
}

\begin{abstract}
This paper develops a fault-tolerant iterative learning control law for a class of linear time-delay differential batch processes with actuator faults using the repetitive process setting. Once the dynamics are expressed in this setting, stability analysis and control law design makes use of the generalized Kalman-Yakubovich-Popov (KYP) lemma in the form of the corresponding linear matrix inequalities (LMIs). In particular, sufficient conditions for the existence of a fault-tolerant control law are developed together with design algorithms for the associated matrices. Under the action of this control law the ILC dynamics have a monotonicity property in terms of an error sequence formed from the difference between the supplied reference trajectory and the outputs produced. An extension to robust control against structured time-varying uncertainties is also developed. Finally, a simulation based case study on the model of a two-stage chemical reactor with delayed recycle is given to demonstrate the feasibility and effectiveness of the new designs.
\end{abstract}

Keywords: Iterative learning control, batch processes, finite frequency range design, fault tolerant control, time-delay systems

\section{1. Introduction}

Iterative learning control (ILC) is a method of iteratively updating the control input to a system that repeats the same task over a finite duration. Each execution is known as a trial, or pass, and the sequence of operations is that a trial is completed, where the finite duration is known as the trial length, the system resets to the starting position and then the next trial can begin, either immediately after resetting is complete or after a further period of time has elapsed. Since the first work, widely credited to [1, ILC has become an established area of control systems research, where the survey papers [2, 3] are one source of the literature up to their years of publication.

In most designs, a reference trajectory is specified and the current trial error is the difference between this signal and the output. The core aim of ILC is to force the sequence formed by the

\footnotetext{
This work is supported by National Natural Science Foundation of China (61273070, 61203092), 111 Project (B12018), the Fundamental Research Funds for the Central Universities (JUSRP51510), the Priority Academic Program Development of Jiangsu Higher Education Institutions and National Science Centre in Poland, grant No. 2014/15/B/ST7/03208

${ }^{*}$ Corresponding author

Email addresses: taohongfeng@hotmail.com (Hongfeng Tao), w.paszke@issi.uz.zgora.pl (Wojciech Paszke), etar@ecs.soton.ac.uk (Eric Rogers), yhz@jiangnan.edu.cn (Huizhong Yang), k.galkowski@issi.uz.zgora.pl (Krzysztof Gałkowski)
} 
errors to converge to zero or to within an acceptable tolerance as measured by the norm on the underlying function space. Moreover, this convergence should be monotonic in the trial number.

In application, an ILC law most often constructs the current trial input as the algebraic sum of the input used on the previous trial and a correction term. The correction term can be designed using data from the complete previous trials or a finite number thereof. A particular feature is the possibility to use non-causal temporal information provided it has been generated on a previous trial.

Since this first work, ILC research has found application in many ares, such as robotic systems, e.g., [4, 5], motion systems, e.g., [6], automotive systems, e.g., [7] and batch processes, e.g., 8], where for this last area the survey paper [9] is a starting point for numerous applications areas in process control. A particular feature of many applications is experimental testing. There has also been an application in robotic-assisted stroke rehabilitation, e.g., [10, 11, with supporting clinical trials. In this last application, the ILC law is used to control the assistive stimulation applied to the relevant muscles in the affected limb as the patient makes repeated attempts at completing a specified finite duration task, e.g., reaching out with the affected arm to an object across a table top. Once an attempt is complete the arm is reset to the starting location and in this time plus a rest period, the control signal for the next attempt can be computed using data collected during the previous attempt. If the patient is improving then as the trial number increases the patients voluntary effort should increase and the applied stimulation decrease. Exactly this feature was detected in the clinical trials.

A common approach to ILC design for discrete dynamics is by a form of lifting. Consider, for simplicity, the single-input single-output (SISO) case where, since the trial length is finite, the input and output on any trial can be represented by super-vectors formed by assembling the values at the sample instants into column vectors. The result is that the ILC dynamics can be represented by a linear matrix difference equation in the error dynamics. Hence tools from discrete linear systems theory can be used to analyze trial-to-trial error convergence and control law design.

Given the finite trial length, trial-to-trial error convergence does not require that the system is stable, i.e., all eigenvalues of the state matrix have modulus strictly less than unity. Of course, there will be consequences for the transient dynamics along the trials in such a case. One solution is to design a feedback control law to stabilize the dynamics and then apply the ILC design to the controlled dynamics. An alternative is to use a 2D systems formulation, i.e., systems that propagate information in two independent directions, which in ILC are from trial-to-trial and along the trial respectively. Early work on this approach includes [12]. Repetitive processes are a particular subclass of $2 \mathrm{D}$ systems have their origins in modeling physical examples [13] for control purposes. These processes are characterized by a series of sweeps, or passes, through dynamics defined over a finite duration. On each pass an output, termed the pass profile, is produced that acts as a forcing function on and hence contributes to the dynamics produced on the next pass.

The repetitive process setting for ILC design has progressed through to experimental verification 4. Design in this setting is a one step process where the control law includes stabilization of the state dynamics on each trial. Also the design methods extend naturally to robust control design where, unlike the lifted setting, matrices formed as the product of nominal state-space model matrices and those from the uncertainty description are always excluded. Moreover, ILC design in this setting transfers directly to differential dynamics, i.e., to cases where design by emulation is the only or preferred setting for analysis and design.

In many industrial processes, time-delays often occur, e.g., in the transmission of material or information between different parts of a system, which, if not compensated, can cause serious deterioration of the stability and performance. Chemical processes are a common industrial source of time-delay systems and there has been research on ILC design for such systems by treating them as differential batch processes over a finite time on each trial. For example, a robust 2D ILC law com- 
bined with the output feedback has been applied to batch processes with state delay and time-varying uncertainties [14].

Industrial control systems usually operate under challenging conditions, which expose the system to faults that, in turn, can cause loss, or serious degradation, of stability and/or performance. Moreover, ILC schemes could be especially sensitive to faults due to the repeated nature of the demand on the control actuator. For such cases, a fault tolerant ILC design is required. Of course, this problem arises in the non-ILC case, see, e.g., 15, where necessary and sufficient conditions for stabilization while retaining a desirable level of the closed-loop performance in the presence of actuator/sensor faults or failures, and also plant-model mismatches, are given.

The design of ILC laws for monotonic trial-to-trial error convergence together with controlled dynamics along the trials, in the SISO case for simplicity, requires frequency attenuation over the complete spectrum. This could be very difficult to enforce in some cases and also in many practical examples, systems properties need only be enforced over finite frequency ranges. Moreover, in other examples it will be required to impose different specifications over finite frequency ranges. One way of solving these problems is to use the generalized Kalman-Yakubovich-Popov (KYP) lemma, see, e.g., [16] for discrete systems with experimental verification in the absence of time delays and no compensation for possible faults. The corresponding results for differential linear systems are given in [17.

This paper develops new results for ILC design applied to differential linear systems with timedelays with the following novel contributions:

- the finite frequency range ILC law design is extended to the fault tolerant control problem for time-delay differential batch processes with actuator faults;

- monotonic trial-to-trial error convergence conditions for the controlled ILC dynamics are derived;

- the extension to robust control against structured uncertainty.

This paper is organized as follows: Section 2 describes a class of linear differential batch processes in the state-space form with actuator faults and a time-delay in the state. Also the ILC design problem is formulated in an equivalent differential linear repetitive process setting. In Section 3 , the corresponding fault tolerant ILC law is designed and sufficient conditions for its existence are developed in terms of generalized KYP lemma and LMIs constraints, which ensure that the nominal and uncertain controlled dynamics are monotonically convergent and stable over a finite frequency range. Section 4 illustrates the feasibility and effectiveness of the new design by a simulation-based application to a two-stage chemical reactor with delayed recycle streams. Finally, the main results are summarized in Section 5 together with some possible areas for further research.

Throughout this paper, the null and identity matrices with the required dimensions are denoted by 0 and $I$, respectively, and the notation $X \prec Y$ (respectively $X \succ Y$ ) is used to represent the negative definite (respectively, positive definite) matrix $X-Y$. The notation $(\star)$ denotes transposed elements in a symmetric matrix and $\rho(\cdot)$ denotes the spectral radius of its matrix argument, i.e., if $\lambda_{i}, 1 \leq i \leq q$, denote the eigenvalues of a $q \times q$ matrix, say $H, \rho(H)=\max _{1 \leq i \leq q}\left|\lambda_{i}\right|$. The symbol $\operatorname{diag}\left\{X_{1}, X_{2}, \cdots, X_{n}\right\}$ denotes a block diagonal matrix with diagonal blocks $X_{1}, X_{2}, \cdots, X_{n}$ and $\operatorname{sym}(\Lambda)=\Lambda+\Lambda^{T}, \otimes$ denotes the Kronecker matrix product, the superscript $*$ denotes the complex conjugate transpose of a matrix and $\mathbb{R}_{+}$denotes the positive real numbers.

The following lemmas are used in the proofs of the main results.

Lemma 1. [18] Given matrices $X, Y, \Phi=\Phi^{T}$ and $\Delta(t)$ of compatible dimensions,

$$
\Phi+X \Delta(t) Y+Y^{T} \Delta^{T}(t) X^{T} \prec 0,
$$


for all $\Delta(t)$ satisfying $\Delta^{T}(t) \Delta(t) \preceq I$ if and only if there exists an $\varepsilon>0$ such that

$$
\Phi+\varepsilon X X^{T}+\varepsilon^{-1} Y^{T} Y \prec 0 .
$$

Lemma 2. [20] Given a symmetric matrix $\Upsilon \in \mathbb{R}^{p \times p}$ and two matrices $\Lambda, \Sigma$ of column dimension $p$, there exists a matrix $W$ such that the $L M I$

$$
\Upsilon+\operatorname{sym}\left\{\Lambda^{T} W \Sigma\right\} \prec 0,
$$

holds if and only if the following two projection inequalities with respect to $W$ are satisfied:

$$
\Lambda^{\perp^{T}} \Upsilon \Lambda^{\perp} \prec 0, \quad \Sigma^{\perp^{T}} \Upsilon \Sigma^{\perp} \prec 0,
$$

where $\Lambda^{\perp}$ and $\Sigma^{\perp}$ are arbitrary matrices whose columns form a basis of the null spaces of $\Lambda$ and $\Sigma$ respectively.

\section{Problem formulation}

\subsection{System description}

Consider a class of differential linear time-invariant batch processes with a single delay and dynamics described in the ILC setting by the following state-space model over $0 \leq t \leq \alpha<\infty, k \geq 0$

$$
\begin{aligned}
\dot{x}_{k}(t) & =A x_{k}(t)+A_{d} x_{k}(t-d)+B u_{k}(t), \\
y_{k}(t) & =C x_{k}(t),
\end{aligned}
$$

where the subscript $k$ denotes the trial number, $\alpha$ is the fixed and finite trial length and $x_{k}(t) \in \mathbb{R}^{n}$, $u_{k}(t) \in \mathbb{R}^{m}$ and $y_{k}(t) \in \mathbb{R}^{p}$ are, respectively, the system state, input and output vectors respectively; $d$ is the unknown time-delay constant satisfying $0<d \leq \bar{d}$, where $\bar{d}$ is a known upper bound. No loss of generality arises from assuming $x_{k}(t)=x_{0, k}, t \in[-d, 0]$ on each trial.

To include actuator faults, write the control input vector as

$$
u_{k}(t)=\left[\begin{array}{lllll}
u_{1, k}(t) & \cdots & u_{i, k}(t) & \cdots & u_{m, k}(t)
\end{array}\right]^{T},
$$

and let

$$
u_{k}^{F}(t)=\left[\begin{array}{lllll}
u_{1, k}^{F}(t) & \cdots & u_{i, k}^{F}(t) & \cdots & u_{m, k}^{F}(t)
\end{array}\right]^{T},
$$

represent the failed actuator with the following fault model, see, e.g. [19],

$$
u_{i, k}^{F}(t)=\Gamma_{i} u_{i, k}(t), i=1,2, \cdots, m,
$$

where

$$
0 \leq \underline{\Gamma}_{i} \leq \Gamma_{i} \leq \bar{\Gamma}_{i}, i=1,2, \cdots, m .
$$

The parameters $\underline{\Gamma}_{i}\left(\underline{\Gamma}_{i} \leq 1\right), \bar{\Gamma}_{i}\left(\bar{\Gamma}_{i} \geq 1\right)$ in this fault model are assumed to be known, i.e., the unknown scalars $\Gamma_{i}$ are assumed to vary within a known range. In particular, $\Gamma_{i}=1$, corresponds to the faultfree case $u_{i}^{F}=u_{i}, \Gamma_{i}=0$ corresponds to a complete failure, $0<\underline{\Gamma}_{i} \leq \Gamma_{i}<1$ and $1<\Gamma_{i} \leq \bar{\Gamma}_{i}$ correspond to partial failures, e.g., partial degradation of an actuator or the abnormal case when the faulty actuator output is larger than the normal controller output. Introduce

$$
\begin{aligned}
& \bar{\Gamma}=\operatorname{diag}\left\{\bar{\Gamma}_{1}, \bar{\Gamma}_{2}, \cdots, \bar{\Gamma}_{m}\right\}, \\
& \underline{\Gamma}=\operatorname{diag}\left\{\underline{\Gamma}_{1}, \underline{\Gamma}_{2}, \cdots, \underline{\Gamma}_{m}\right\}, \\
& \Gamma=\operatorname{diag}\left\{\Gamma_{1}, \Gamma_{2}, \cdots, \Gamma_{m}\right\},
\end{aligned}
$$


and

$$
\begin{aligned}
q & =\operatorname{diag}\left\{q_{1}, q_{2}, \cdots, q_{m}\right\}, \\
q_{0} & =\operatorname{diag}\left\{q_{10}, q_{20}, \cdots, q_{m 0}\right\}, \\
q_{i} & =\frac{\left(\bar{\Gamma}_{i}+\underline{\Gamma}_{i}\right)}{2}, q_{i 0}=\frac{\bar{\Gamma}_{i}-\underline{\Gamma}_{i}}{\bar{\Gamma}_{i}+\underline{\Gamma}_{i}} .
\end{aligned}
$$

Introducing the notation

$$
\begin{aligned}
\Gamma_{0} & =\operatorname{diag}\left\{\Gamma_{01}, \Gamma_{02}, \cdots, \Gamma_{0 m}\right\}, \\
\left|\Gamma_{0}\right| & =\operatorname{diag}\left\{\left|\Gamma_{01}\right|,\left|\Gamma_{02}\right|, \cdots,\left|\Gamma_{0 m}\right|\right\}, \\
\Gamma_{0 i} & =\left(\Gamma_{i}-q_{i}\right) / q_{i},
\end{aligned}
$$

and using (5) and (6), $\Gamma$ can be written as

$$
\Gamma=\left(I+\Gamma_{0}\right) q,
$$

where

$$
\left|\Gamma_{0}\right| \preceq q_{0} \preceq I .
$$

In this paper it is assumed that the upper and lower bounds on the fault range of each actuator fault are known, i.e., $\underline{\Gamma}_{i}$ and $\bar{\Gamma}_{i}$. Hence an additional parameters $q_{i}$, as the entries in the vector $q$ in (6), can be used to scale the original range of the unknown scalars $\Gamma_{i}$ such that $\Gamma$ in (5) can be treated as a structured uncertainty of form (7) with known and unknown vectors $q_{0}$ and $\Gamma_{0}$ respectively. This model (7) for faults is very useful since the unknown vector $\Gamma_{0}$ can be eliminated by application of Lemma 1 and hence it is possible to cover a wide range of vectors $\Gamma$ and obtain less conservative conditions than previously reported designs in this area. In comparison to the well known polytopic form (7), the number of LMIs required will be lower since in the latter each vertex must be considered. Hence for analysis and design, (4) with the actuator faults model included is replaced by

$$
\begin{aligned}
\dot{x}_{k}(t) & =A x_{k}(t)+A_{d} x_{k}(t-d)+B \Gamma u_{k}(t), \\
y_{k}(t) & =C x_{k}(t) .
\end{aligned}
$$

The design of the ILC law can now proceed in one of two general settings. The first is to sample the dynamics and then apply the lifting approach, i.e., since the trial length is finite a column vector of, e.g., the input, can be constructed where, in the SISO case for ease of presentation, the entries are the values at the sampling instants along the trial. Repeating this step for the output enables the ILC trial-to-trial error dynamics to be described in terms of a linear difference equation in the trial number $k$. The basic ILC design problem then is to ensure that trial-to-trial error convergence occurs to zero or 'sufficiently close' as measured by an appropriate norm and standard systems theory can be used.

Given that the trial length is finite, trial-to-trial error convergence can be enforced for systems that are unstable, which, of course, produces unacceptable dynamics along the trials since the trial length is finite and the along trial dynamics are bounded but not uniformly. If this behavior is unacceptable the lifted model based design requires that a stabilizing control law is first designed and implemented and then ILC applied to the resulting dynamics. This is a two-step design procedure for which an alternative is to use a 2D systems setting where one direction of information is from trial-to-trial and the other along the trial.

Repetitive processes are a particular class of 2D systems where a series of sweeps are made through a set of dynamics defined over a finite duration. Once each sweep is completed, the dynamics reset to 
the starting location and the next sweep is made. On any sweep, the output on the previous sweep explicitly contributes to the current sweep output and hence the link to ILC dynamics.

Repetitive processes are a natural setting for ILC design and previous results have been experimentally validated. This setting is a one-step design and can be extended to robust control. Moreover, it extends naturally to cases where design in the differential domain and then sampling for implementation is the preferred or only option. Next the representation of differential repetitive processes and the associated stability theory are given as background to the new ILC design in this paper.

\subsection{ILC as a repetitive process}

The state-space model of a differential linear repetitive process [13] over the finite pass length $\alpha$ is

$$
\begin{aligned}
\dot{x}_{k+1}(t) & =\mathcal{A} x_{k+1}(t)+\mathcal{B} u_{k+1}(t)+\mathcal{B}_{0} y_{k}(t), \\
y_{k+1}(t) & =\mathcal{C} x_{k+1}(t)+\mathcal{D} u_{k+1}(t)+\mathcal{D}_{0} y_{k}(t),
\end{aligned}
$$

where, on pass $k, x_{k}(t) \in \mathbb{R}^{n}, u_{k}(t) \in \mathbb{R}^{m}$ and $y_{k}(t) \in \mathbb{R}^{p}$ are respectively, the process state, input and pass profile (output) vectors. To complete the process description it is necessary to specify the boundary conditions, i.e., the pass state initial vector sequence $x_{k}(0), k \geq 1$, and the initial pass profile $y_{0}(t)$. In this paper, the initial state vector on each pass is taken as the zero vector and the initial pass profile vector entries are assumed to be specified over the pass length as known functions of $t$.

In $(10), \mathcal{B}_{0} y_{k}(t)$ and $\mathcal{D}_{0} y_{k}(t)$ represent the contributions of the previous pass profile vector to the current pass state and pass profile vectors respectively. This inter-pass interaction is the source of the unique control problem for these processes where the sequence of pass profiles $\left\{y_{k}\right\}_{k \geq 1}$ can contain oscillations that increase in amplitude from pass-to-pass, i.e., with increasing $k$. Such behavior cannot be regulated by the application of standard linear systems control action and this has motivated the development of a stability theory and control law design algorithms, which is an ongoing area of research.

The stability theory [13] for these processes splits into the properties of asymptotic stability and stability along the pass respectively. Asymptotic stability guarantees a bounded sequence of pass profiles (i.e. output signals) for a bounded initial pass profile over the finite and fixed pass length $\alpha$, whereas stability along the pass is stronger since it requires this property uniformly, i.e., for all possible values of the pass length. Asymptotic stability is a necessary condition for stability along the pass. To conform with the ILC literature, the terms pass is replaced by trial in the remainder of this paper.

Stability along the trial of processes described by $(10)$ is characterized by the following result.

Lemma 3. [13] A differential linear repetitive process described by (10) is stable along the trial if and only if

i) $\rho\left(\mathcal{D}_{0}\right)<1$,

ii) all eigenvalues of the matrix $\mathcal{A}$ have strictly negative real parts, and,

iii) all eigenvalues of $G(j \omega)=\mathcal{C}(j \omega I-\mathcal{A})^{-1} \mathcal{B}_{0}+\mathcal{D}_{0}$, have modulus strictly less than unity $\forall$ real frequencies $\omega$.

The first condition in the above Lemma is the necessary and sufficient condition for asymptotic stability. This condition is independent of the along the trial dynamics and holds even if $\mathcal{A}$ has eigenvalues with real parts greater than or equal to zero. Even if this matrix is required to be stable 
in the standard linear systems sense then this does not guarantee stability along the trial, a counterexample to this claim is given in 13. To achieve stability along the trial also requires condition iii) and this condition requires that each frequency component of the initial trial profile is attenuated from trial-to-trial.

To formulate the ILC design problem in the repetitive process setting, consider a system described (9) and a ILC law that constructs the current trial input as that used on the previous trial plus a corrective term, i.e., a law of the form

$$
u_{k}(t)=u_{k-1}(t)+r_{k}(t),
$$

where $r_{k}(t)$ is the correction term computed using an algorithm that makes use of previous trial data. Given the reference signal $y_{d}(t)$, the error on trial $k$ is

$$
e_{k}(t)=y_{d}(t)-y_{k}(t),
$$

and introduce for analysis purposes only the vector

$$
\eta_{k}(t)=\int_{0}^{t}\left[x_{k}(\tau)-x_{k-1}(\tau)\right] \mathrm{d} \tau .
$$

Without loss of generality, it is assumed that $y_{d}(0)=y_{k}(0)=C x_{k}(0)$ and, due to the initial conditions assumed for (4), $\eta_{k}(0)=0$. Hence

$$
\dot{\eta}_{k}(t)=\int_{0}^{t}\left[\dot{x}_{k}(\tau)-\dot{x}_{k-1}(\tau)\right] \mathrm{d} \tau=A \eta_{k}(t)+A_{d} \eta_{k}(t-d)+B \Gamma \int_{0}^{t} r_{k}(\tau) \mathrm{d} \tau,
$$

and

$$
e_{k}(t)-e_{k-1}(t)=-C\left[x_{k}(t)-x_{k-1}(t)\right]=-C A \eta_{k}(t)-C A_{d} \eta_{k}(t-d)-C B \Gamma \int_{0}^{t} r_{k}(\tau) \mathrm{d} \tau .
$$

Suppose that the modification term in the ILC law (11) takes the form

$$
r_{k}(t)=K_{1} \dot{\eta}_{k}(t)+K_{2} \dot{e}_{k-1}(t),
$$

where $K_{1}$ and $K_{2}$ are the control law matrices to be determined. This control law correction term is the sum of state feedback control based on $\eta_{k}(t)$ plus a feedforward term based on the previous trial error $\left(e_{k-1}\right)$. Moreover, by (11)-(16) the controlled ILC dynamics can be written as a differential repetitive process with time-delays as

$$
\begin{aligned}
\dot{\eta}_{k}(t) & =\mathcal{A} \eta_{k}(t)+A_{d} \eta_{k}(t-d)+\mathcal{B}_{0} e_{k-1}(t), \\
e_{k}(t) & =\mathcal{C} \eta_{k}(t)+C_{d} \eta_{k}(t-d)+\mathcal{D}_{0} e_{k-1}(t),
\end{aligned}
$$

where

$$
\begin{aligned}
\mathcal{A} & =A+B \Gamma K_{1}, \mathcal{C}=-C\left(A+B \Gamma K_{1}\right)=-C \mathcal{A}, \\
C_{d} & =-C A_{d}, \mathcal{B}_{0}=B \Gamma K_{2}, \mathcal{D}_{0}=I-C B \Gamma K_{2} .
\end{aligned}
$$

The repetitive process stability theory [13] applies to this case and gives, on applying Lemma 3, the following necessary and sufficient conditions for stability along the trial of the controlled dynamics.

Lemma 4. A differential linear repetitive process described by $(17)$ is stable along the trial $\forall d \in[0, \bar{d}]$ if and only if 
i) $\rho\left(\mathcal{D}_{0}\right)<1$,

ii) all eigenvalues of the matrix $\left(\mathcal{A}+e^{-j \omega d} A_{d}\right)$ have strictly negative real parts $\forall \omega \in \mathbb{R}_{+} \cup \infty$ and $d \in[0, \bar{d}]$,

iii) all eigenvalues of $G(j \omega)=\left(\mathcal{C}+e^{-j \omega d} C_{d}\right)\left(j \omega I-\mathcal{A}-e^{-j \omega d} A_{d}\right)^{-1} \mathcal{B}_{0}+\mathcal{D}_{0}$ have modulus strictly less than unity $\forall \omega \in \mathbb{R}_{+} \cup \infty$ and $d \in[0, \bar{d}]$.

The last condition in this result requires frequency attenuation of the previous trial error over the complete spectrum for any delay $d \in[0, \bar{d}]$, a point returned to later in this paper.

Remark 1. It is important to stress that the repetitive process dynamics, i.e., where the previous trial output affects the current trial output arises only from the application of the ILC law. Hence the analysis in this paper is not relevant to other batch processes where such a property is not present.

\section{Design of a fault tolerant controller over a finite frequency domain}

Applying the Laplace transform to (17) (see [13] and the cited references on how detrimental effects due to the finite trial length can be avoided) gives

$$
E_{k+1}(s)=G(s) E_{k}(s),
$$

with

$$
G(j \omega)=\left.G(s)\right|_{s=j \omega}=\left(\mathcal{C}+e^{-j \omega d} C_{d}\right)\left(j \omega I-\mathcal{A}-e^{-j \omega d} A_{d}\right)^{-1} \mathcal{B}_{0}+\mathcal{D}_{0} .
$$

Hence the tracking error converges as $k \rightarrow \infty$ if and only if

$$
\rho(G(j \omega))<1, \forall \omega \in \mathbb{R}_{+} \cup \infty .
$$

The last inequality can be replaced by the requirement that there exists a Hermitian matrix $\mathcal{P}(j \omega) \succ 0$ such that

$$
G(j \omega)^{*} \mathcal{P}(j \omega) G(j \omega)-\mathcal{P}(j \omega) \prec 0, \forall \omega \in \mathbb{R}_{+} \cup \infty,
$$

but the dependence of $\mathcal{P}(j \omega)$ on $\omega$ is unknown. One possible approach to solve this problem is to use the same constant matrix $\forall \omega \in \mathbb{R}_{+} \cup \infty$.

Lemma 5. Consider a differential repetitive process described by (17) with corresponding transferfunction matrix (19). Then (20) holds if there exists $\mathcal{P} \succ 0$ such that

$$
\left[\begin{array}{c}
G(j \omega) \\
I
\end{array}\right]^{*}\left[\begin{array}{cc}
\mathcal{P} & 0 \\
0 & -\mathcal{P}
\end{array}\right]\left[\begin{array}{c}
G(j \omega) \\
I
\end{array}\right] \prec 0, \forall \omega \in \mathbb{R}_{+} \cup \infty .
$$

Proof. Omitted since the result follows from direct application of Lyapunov stability theory for standard linear systems.

Remark 2. As shown in 21 the use of real symmetric matrix $\mathcal{P}$ instead of a Hermitian matrix introduces no additional conservatism and hence the computational load is reduced.

It is known, see, e.g. 22, that some ILC laws exhibit poor transients during the convergence process even if (20) is satisfied. In particular, the tracking error may grow over the initial trials before converging as $k \rightarrow \infty$. To avoid this problem, a stronger convergence criteria is required and, in particular, monotonic trial-to-trial error convergence holds if

$$
\bar{\sigma}(G(j \omega))<1, \omega \in \mathbb{R}_{+} \cup \infty
$$


where $\bar{\sigma}(\cdot)$ denotes the maximum singular value of its matrix argument. In common with much of the ILC literature (22) is used from this point onwards. Moreover, since

$$
\bar{\sigma}(G(j \omega))<1 \Leftrightarrow\|G(j \omega)\|_{\infty}<1, \omega \in \mathbb{R}_{+} \cup \infty,
$$

then (22) implies that

$$
\left\|e_{k+1}(t)\right\|_{2} \leq\|G(s)\|_{\infty}\left\|e_{k}(t)\right\|_{2},
$$

where $\|\cdot\|_{2}$ denotes the $L_{2}$ norm. Hence, it is obvious that

$$
\left\|e_{k}(t)\right\|_{2} \leq\|G(s)\|_{\infty}^{k}\left\|e_{0}(t)\right\|_{2},
$$

and therefore if

$$
\|G(j \omega)\|_{\infty}<1, \omega \in \mathbb{R}_{+} \cup \infty,
$$

then monotonic trial-to-trial error convergence occurs in $L_{2}$. Additionally, (22) holds provided (21) is feasible for $\mathcal{P}=I$.

In applications, it may be required to impose different frequency constraints over finite frequency ranges, e.g., over a low range of frequencies, depending on the application considered. Moreover, at least finite frequency ranges for any disturbances present can often be obtained for physical examples. The stability theory of Lemma 4, or equivalents, imposes the same condition over the complete frequency spectrum and this may be conservative in some cases. An alternative whereby different specifications can be specified over different frequency ranges is possible as developed in the remainder of this paper using the generalized KYP lemma.

One practically relevant case would be where it is only required to impose stability along the trial over a finite frequency range, i.e., $\|G(j \omega)\|_{\infty}<1, \forall \omega \in \Omega$, where $\Omega$ denotes the finite frequency range of interest. Hence each design specification should be given not for the entire frequency range but for a certain frequency range of relevance. Thus a set of specifications would generally consist of different requirements in various frequency ranges. One way of dealing with this case is to modify (23) to $\|G(j \omega)\|_{\infty}<1, \forall \omega \in \Omega$, where $\Omega$ is a finite frequency range of interest. In general, it could be required to impose different specifications over a low frequency range specified by $|\omega| \leq \varpi_{l}$, a middle frequency range specified by $\varpi_{1} \leq \omega \leq \varpi_{2}$ and a high frequency range specified by $|\omega| \geq \varpi_{h}$, where the frequency limits are application dependent. See [16 for results with experimental verification for discrete systems without time delays and fault free.

The analysis in the remainder of this paper makes use of the following result, which is Theorem 1 in 23 for the single delay case.

Lemma 6. [23] For a given real symmetric matrix $\Pi$ of compatible dimensions and any delay $d$ satisfying $0<d \leq \bar{d}$, a transfer-function matrix $G(j \omega)$ defined by 19 satisfies the condition of (20) over a specified frequency range $\Omega$ if there exist $P \succ 0, Z \succ 0, Q \succ 0$ and $X \succ 0$, such that

$$
\begin{aligned}
& {\left[\begin{array}{ccc}
\mathcal{A} & A_{d} & \mathcal{B}_{0} \\
I & 0 & 0
\end{array}\right]^{T}\left(\Phi \otimes P+\Psi \otimes Q+\Psi_{0} \otimes \bar{d} Z\right)\left[\begin{array}{ccc}
\mathcal{A} & A_{d} & \mathcal{B}_{0} \\
I & 0 & 0
\end{array}\right]} \\
& +\left[\begin{array}{ccc}
\mathcal{C} & C_{d} & \mathcal{D}_{0} \\
0 & 0 & I
\end{array}\right]^{T} \Pi\left[\begin{array}{ccc}
\mathcal{C} & C_{d} & \mathcal{D}_{0} \\
0 & 0 & I
\end{array}\right]+\left[\begin{array}{ccc}
X-\bar{d}^{-1} Z & \bar{d}^{-1} Z & 0 \\
\bar{d}^{-1} Z & -X-\bar{d}^{-1} Z & 0 \\
0 & 0 & 0
\end{array}\right] \prec 0 .
\end{aligned}
$$

Moreover, the following frequency domain inequality also holds

$$
\left[\begin{array}{c}
G(j \omega) \\
I
\end{array}\right]^{*} \Pi\left[\begin{array}{c}
G(j \omega) \\
I
\end{array}\right] \prec 0, \forall \omega \in \Omega
$$


where

$$
\Phi=\left[\begin{array}{ll}
0 & I \\
I & 0
\end{array}\right], \Psi_{0}=\left[\begin{array}{ll}
I & 0 \\
0 & 0
\end{array}\right],
$$

and $\Omega$ is specified by the following choices for $\Psi$

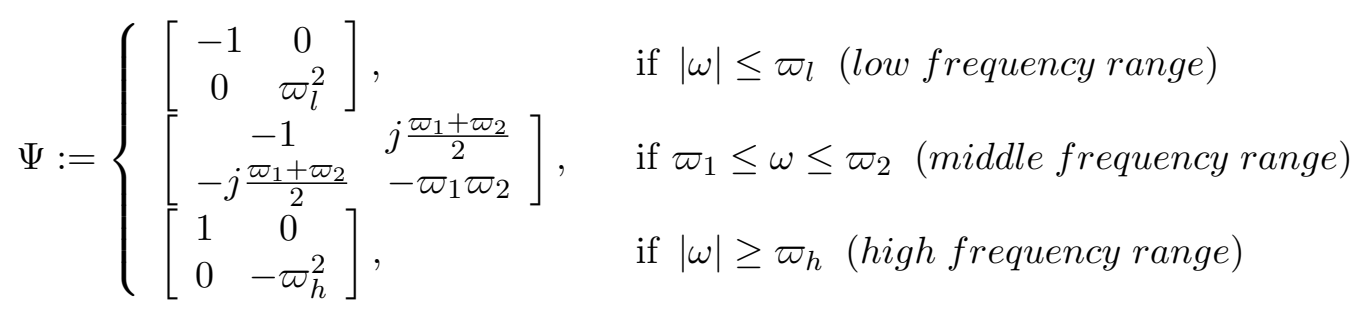

Remark 3. This last result requires $P \succ 0$ and $X \succ 0$ to guarantee that all eigenvalues of the matrix $\left(\mathcal{A}+e^{-j \omega d} A_{d}\right)$ have strictly negative real parts [23].

The inequality conditions of Lemma 6 are not convex and to obtain LMI conditions on which to base control law design further development (not given in [23]) is required, based on decoupling the repetitive process state-space model matrices using matrix variables arising from the generalized KYP Lemma and the projection lemma (Lemma 2). To proceed set $\Pi=\operatorname{diag}\{I,-I\}$ in (24) and then (25) implies that (22) is satisfied for the different frequency ranges defined by the matrix $\Psi$. Next, introduce

$$
\Upsilon=\left[\begin{array}{ll}
I & 0 \\
0 & I \\
0 & 0 \\
0 & 0
\end{array}\right] \Xi\left[\begin{array}{ll}
I & 0 \\
0 & I \\
0 & 0 \\
0 & 0
\end{array}\right]^{T}+\left[\begin{array}{cc}
0 & 0 \\
\mathcal{C}^{T} & 0 \\
C_{d}^{T} & 0 \\
\mathcal{D}_{0}^{T} & I
\end{array}\right] \Pi\left[\begin{array}{cc}
0 & 0 \\
\mathcal{C}^{T} & 0 \\
C_{d}^{T} & 0 \\
\mathcal{D}_{0}^{T} & I
\end{array}\right]^{T}+\left[\begin{array}{cccc}
0 & 0 & 0 & 0 \\
0 & X-\bar{d}^{-1} Z & \bar{d}^{-1} Z & 0 \\
0 & \bar{d}^{-1} Z & -X-\bar{d}^{-1} Z & 0 \\
0 & 0 & 0 & 0
\end{array}\right]
$$

where $\Xi=\left(\Phi \otimes P+\Psi \otimes Q+\Psi_{0} \otimes \bar{d} Z\right)$ is the only matrix whose block entries depend on chosen frequency range, i.e., low, middle or high. Also the matrix $\Xi$ can be partitioned as

$$
\Xi=\Phi \otimes P+\Psi \otimes Q+\Psi_{0} \otimes \bar{d} Z=\left[\begin{array}{c|c}
\Xi_{1} & \Xi_{2} \\
\hline(\star) & \Xi_{3}
\end{array}\right],
$$

where

- for the low frequency range

$$
\Xi=\left[\begin{array}{c|c}
\Xi_{1} & \Xi_{2} \\
\hline(\star) & \Xi_{3}
\end{array}\right]=\left[\begin{array}{c|c}
-Q+\bar{d} Z & P \\
\hline(\star) & \varpi_{l}^{2} Q
\end{array}\right],
$$

- for the middle frequency range

$$
\Xi=\left[\begin{array}{c|c}
\Xi_{1} & \Xi_{2} \\
\hline(\star) & \Xi_{3}
\end{array}\right]=\left[\begin{array}{c|c}
-Q+\bar{d} Z & P+j \frac{\varpi_{1}+\varpi_{2}}{2} Q \\
\hline(\star) & -\varpi_{1} \varpi_{2} Q
\end{array}\right],
$$

- and for the high frequency range

$$
\Xi=\left[\begin{array}{c|c}
\Xi_{1} & \Xi_{2} \\
\hline(\star) & \Xi_{3}
\end{array}\right]=\left[\begin{array}{c|c}
Q+\bar{d} Z & P \\
\hline(\star) & -\varpi_{h}^{2} Q
\end{array}\right],
$$


and $(24)$ can be rewritten as

$$
\left[\begin{array}{ccc}
\mathcal{A} & A_{d} & \mathcal{B}_{0} \\
I & 0 & 0 \\
0 & I & 0 \\
0 & 0 & I
\end{array}\right]^{T} \Upsilon\left[\begin{array}{ccc}
\mathcal{A} & A_{d} & \mathcal{B}_{0} \\
I & 0 & 0 \\
0 & I & 0 \\
0 & 0 & I
\end{array}\right] \prec 0
$$

This last inequality is of the form of the first of those in (3) where

$$
\Lambda^{\perp}=\left[\begin{array}{ccc}
\mathcal{A} & A_{d} & \mathcal{B}_{0} \\
I & 0 & 0 \\
0 & I & 0 \\
0 & 0 & I
\end{array}\right]
$$

and for this $\Lambda^{\perp}, \Lambda=\left[\begin{array}{cccc}-I & \mathcal{A} & A_{d} & \mathcal{B}_{0}\end{array}\right]$. Moreover, to use the result of Lemma 2 it is required to find $\Sigma^{\perp}$ that satisfies the second inequality of (3). Choosing

$$
\Sigma=\left[\begin{array}{llll}
\beta I & I & 0 & 0
\end{array}\right],
$$

gives

$$
\Sigma^{\perp}=\left[\begin{array}{ccc}
I & 0 & 0 \\
-\beta I & 0 & 0 \\
0 & I & 0 \\
0 & 0 & I
\end{array}\right]
$$

where $\beta$ is a given scalar and the desired formulations for low/middle/high frequency range are obtained by selecting $\beta$ as:

- $\beta=0$ for low and middle frequency range,

- $\beta>0$ for high frequency range.

The above analysis introduces a slightly different formulation for the high frequency range since $\Xi_{1}$ in (30) cannot be negative definite because $Q$ and $Z$ are positive definite. Also with the $\Sigma$ and $\Sigma^{\perp}$ given above, the second inequality in (3) becomes

$$
\Sigma^{\perp^{T}} \Upsilon \Sigma^{\perp}=\left[\begin{array}{ccc}
\Xi_{1}-\beta^{2}\left(\Xi_{3}-\mathcal{C}^{T} \mathcal{C}-X+\bar{d} Z\right)-\beta\left(\Xi_{2}+\Xi_{2}^{T}\right) & -\beta \mathcal{C}^{T} C_{d}-\beta \bar{d} Z & -\beta \mathcal{C}^{T} \mathcal{D}_{0} \\
(\star) & C_{d}^{T} C_{d}-X-\bar{d}^{-1} Z & C_{d}^{T} \mathcal{D}_{0} \\
(\star) & (\star) & \mathcal{D}_{0}^{T} \mathcal{D}_{0}-I
\end{array}\right] \prec 0
$$

The first two new results in this paper can now be derived where, unlike Theorem 1 in [23], the high frequency range is considered separately since it requires $\beta>0$ (not $\beta=0$ ) and hence the resulting LMI is more complex.

Theorem 1. An ILC scheme described as a differential linear repetitive process of the form (17) under an actuator fault of the form (5)-(8) is stable along the trial and hence monotonic trial-to-trial error convergence occurs for the performance specifications over low and middle frequency ranges given in (25) and any delay $d$ satisfying $0<d \leq \bar{d}$ if there exist matrices $\hat{P} \succ 0, \hat{Q} \succ 0, \hat{X} \succ 0$, $\hat{Z} \succ 0, S, L$ and a scalar $\varepsilon_{1}>0$, such that

$$
\Theta_{11}=\left[\begin{array}{cc}
\Theta_{1} & \Theta_{2} \\
(\star) & \Theta_{3}
\end{array}\right] \prec 0
$$


where

$$
\Theta_{1}=\left[\begin{array}{cccc}
\hat{\Xi}_{1} & \hat{\Xi}_{2}-S^{T} & 0 & 0 \\
(\star) & \mathrm{T}_{1} & \mathrm{~T}_{2} & q B L \\
(\star) & (\star) & \mathrm{T}_{3} & 0 \\
(\star) & (\star) & (\star) & -I
\end{array}\right], \Theta_{2}=\left[\begin{array}{ccc}
0 & 0 & 0 \\
\mathrm{~T}_{4} & \varepsilon_{1} q_{0} B & \left(q X_{1}\right)^{T} \\
\mathrm{~T}_{5} & 0 & 0 \\
\mathrm{~T}_{6} & 0 & q L^{T}
\end{array}\right], \Theta_{3}=\left[\begin{array}{ccc}
-I & -\varepsilon_{1} q_{0} C B & 0 \\
(\star) & -\varepsilon_{1} I & 0 \\
(\star) & (\star) & -\varepsilon_{1} I
\end{array}\right],
$$

315 and

$$
\begin{aligned}
S & =W^{-1}, \hat{\Xi}_{1}=S^{T} \Xi_{1} S, \hat{\Xi}_{2}=S^{T} \Xi_{2} S, \hat{\Xi}_{3}=S^{T} \Xi_{3} S, \hat{X}=S^{T} X S, \hat{Z}=S^{T} Z S, \hat{Q}=S^{T} Q S, \\
\mathrm{~T}_{1} & =\hat{\Xi}_{3}+\hat{X}-\bar{d}^{-1} \hat{Z}+\operatorname{sym}\left\{A S+q B X_{1}\right\}, \mathrm{T}_{2}=A_{d} S+\bar{d}^{-1} \hat{Z}, \mathrm{~T}_{3}=-\hat{X}-\bar{d}^{-1} \hat{Z}, \\
\mathrm{~T}_{4} & =-\left(C A S+q C B X_{1}\right)^{T}, \mathrm{~T}_{5}=-\left(C A_{d} S\right)^{T}, \mathrm{~T}_{6}=(I-q C B L)^{T}, \hat{P}=S^{T} P S .
\end{aligned}
$$

316

If this LMI is feasible, the corresponding matrices in the control law (16) are given by

$$
K_{1}=X_{1} S^{-1}, K_{2}=L
$$

317 Proof. Suppose that there exist $\hat{P} \succ 0, \hat{Q} \succ 0, \hat{X} \succ 0, \hat{Z} \succ 0, S, L$ and a scalar $\varepsilon_{1}>0$, such that 318

the LMI (33) is feasible. Then application of the Schur's complement formula to (33) gives

$$
\Phi_{s}+\varepsilon_{1} q_{0}^{2} H_{1} H_{1}^{T}+\varepsilon_{1}^{-1} F_{1}^{T} F_{1} \prec 0,
$$

319 where

$$
\Phi_{s}=\left[\begin{array}{ccccc}
\hat{\Xi}_{1} & \hat{\Xi}_{2}-S^{T} & 0 & 0 & 0 \\
(\star) & \mathrm{T}_{1} & \mathrm{~T}_{2} & q B L & \mathrm{~T}_{4} \\
(\star) & (\star) & \mathrm{T}_{3} & 0 & \mathrm{~T}_{5} \\
(\star) & (\star) & (\star) & -I & \mathrm{~T}_{6} \\
(\star) & (\star) & (\star) & (\star) & -I
\end{array}\right], H_{1}=\left[\begin{array}{c}
0 \\
B \\
0 \\
0 \\
-C B
\end{array}\right], F_{1}=\left[\begin{array}{lllll}
0 & q X_{1} & 0 & q L & 0
\end{array}\right] .
$$

Based on (8) and applying Lemma 1, this last inequality yields

$$
\Phi_{s}+\operatorname{sym}\left\{H_{1} \Gamma_{0} F_{1}\right\} \prec 0 .
$$

321 Next, by introducing $\widehat{\Xi}_{3}=\hat{\Xi}_{3}+\hat{X}-\bar{d}^{-1} \hat{Z}+\operatorname{sym}\{\mathcal{A} S\}$, this last inequality can be rewritten as

$$
\left[\begin{array}{ccccc}
\hat{\Xi}_{1} & \hat{\Xi}_{2}-S^{T} & 0 & 0 & 0 \\
(\star) & \widehat{\Xi}_{3} & \bar{d}^{-1} \hat{Z}+A_{d} S & \mathcal{B}_{0} & S^{T} \mathcal{C}^{T} \\
(\star) & (\star) & -\hat{X}-\bar{d}^{-1} \hat{Z} & 0 & S^{T} C_{d}^{T} \\
(\star) & (\star) & (\star) & -I & \mathcal{D}_{0}^{T} \\
(\star) & (\star) & (\star) & (\star) & -I
\end{array}\right] \prec 0,
$$

322 and pre- and post-multiplying this last inequality by $\operatorname{diag}\left\{S^{-T}, S^{-T}, S^{-T}, I, I\right\}$ and its transpose, 323 respectively, gives 
where $\tilde{\Xi}_{3}=\Xi_{3}+X-\bar{d}^{-1} Z+\operatorname{sym}\left\{\mathcal{A}^{T} W\right\}$. Another application of the Schur's complement formula gives that (36) holds if and only if

$$
\left[\begin{array}{cccc}
\Xi_{1} & \Xi_{2}-W & 0 & 0 \\
(\star) & \tilde{\Xi}_{3}+\mathcal{C}^{T} \mathcal{C} & \mathcal{C}^{T} C_{d}+\bar{d}^{-1} Z+W^{T} A_{d} & \mathcal{C}^{T} \mathcal{D}_{0}+W^{T} \mathcal{B}_{0} \\
(\star) & (\star) & C_{d}^{T} C_{d}-X-\bar{d}^{-1} Z & C_{d}^{T} \mathcal{D}_{0} \\
(\star) & (\star) & (\star) & \mathcal{D}_{0}^{T} \mathcal{D}_{0}-I
\end{array}\right] \prec 0 .
$$

Clearly, feasibility of this last inequality implies that $\mathcal{D}_{0}^{T} \mathcal{D}_{0}-I \prec 0$ and therefore the condition $i$ ) of Lemma 4 must hold. Moreover, the last inequality can be rewritten as

$$
\Upsilon+\operatorname{sym}\left\{\Lambda^{T} W \Sigma\right\} \prec 0,
$$

where $\Upsilon$ is defined in (26) and $\Lambda=\left[\begin{array}{llll}-I & \mathcal{A} & A_{d} & \mathcal{B}_{0}\end{array}\right], \Sigma=\left[\begin{array}{llll}0 & I & 0 & 0\end{array}\right]$. Moreover, $\Sigma=$ $\left[\begin{array}{llll}0 & I & 0 & 0\end{array}\right]$ is obtained by setting $\beta=0$ in (31). Therefore, by Lemma 2 it follows that (37) is feasible if and only if (24) holds. Hence conditions ii) and iii) of Lemma 4 must hold and the proof is complete.

Selecting $\beta>0$ and using a similar approach gives the following result for control law design in the high-frequency range.

Theorem 2. With the notation of (34), an ILC scheme described as a differential linear repetitive process of the form (17) under an actuator fault of the form (5)-(8) is stable along the trial and hence monotonic trial-to-trial error convergence occurs for performance specifications over the high frequency range (25) and any delay d satisfying $0<d \leq \bar{d}$ if there exist $\hat{P} \succ 0, \hat{Q} \succ 0, \hat{X} \succ 0, \hat{Z} \succ 0$, matrices $S, L$ and positive scalars $\beta, \varepsilon_{2}$ such that

$$
\Theta_{12}=\left[\begin{array}{ccccccc}
\hat{\Xi}_{1}-\beta S-\beta S^{T} & \hat{\Xi}_{2}-S^{T}+A S+q B X_{1} & \beta A_{d} S & \beta q B L & 0 & 0 & 0 \\
(\star) & \mathrm{T}_{1} & \mathrm{~T}_{2} & q B L & \mathrm{~T}_{4} & \varepsilon_{2} q_{0} B & \left(q X_{1}\right)^{T} \\
(\star) & (\star) & \mathrm{T}_{3} & 0 & \mathrm{~T}_{5} & 0 & 0 \\
(\star & (\star) & (\star) & -I & \mathrm{~T}_{6} & 0 & q L^{T} \\
(\star) & (\star) & (\star) & (\star) & -I & -\varepsilon_{2} q_{0} C B & 0 \\
(\star) & (\star) & (\star) & (\star) & (\star) & -\varepsilon_{2} I & 0 \\
(\star) & (\star) & (\star) & (\star) & (\star) & (\star) & -\varepsilon_{2} I
\end{array}\right] \prec 0,
$$

If this LMI is feasible, the corresponding matrices in the control law (16) are given by (35).

Proof. This follows from routine changes to the proof of the previous result to account for $\beta>0$. Hence the details are omitted.

\subsection{Practical implementation issues}

In applications terms a critical problem is to achieve the desired shape of $\bar{\sigma}(G(j \omega))$ over the complete frequency range to account for the spectra of exogenous signals, to penalize regulated variables and to specify the level of plant model uncertainty. In particular, the possibility to impose different performance specifications has considerable practical significance since common performance issues occur over different frequency ranges. For example, trial-to-trial error convergence rate is in the 'low' frequency range whereas low sensitivity to disturbances and sensor noise are in 'high' frequency range. To impose different performance specifications in different frequency ranges, divide the entire frequency range, i.e., from $\omega=0$ to $\omega=\infty$, into $H$ intervals (not necessarily containing the same number of frequencies) such that

$$
[0, \infty)=\bigcup_{h=1}^{H}\left[\omega_{h-1}, \omega_{h}\right]
$$


where $\omega_{0}=0$ and $\omega_{H}=\infty$. Then the LMI conditions in Theorems 1 and 2, respectively, can be applied over these frequency intervals. In particular, the control performance for low frequency range is imposed over the first interval, i.e. $h=1$ and those for the high frequency range over the last interval, i.e. $h=H$. The specifications for the middle frequency range can be defined over remaining intervals for $2 \leq h \leq H-1$. Furthermore, the LMI condition in Theorems 1 and 2 guarantee that $\bar{\sigma}(G(j \omega))<1$ (where $G(j \omega)$ is defined in $(19)$ ) over the prescribed frequency ranges. However, some practical control specifications require that $\bar{\sigma}(G(j \omega))<\mu$ where $0<\mu \leq 1$. This means that the prescribed level of attenuation of some frequencies is required. For instance, by minimizing $\mu$ in the low frequency range a higher speed of monotonic trial-to-trial error convergence is obtained. These specifications can be easily included in the LMI conditions of Theorems 1 and 2, respectively, by replacing the term $-I$ by $-\mu^{2} I$ in block $(4,4)$ of $\Theta_{1}$ in $(33)$ and block $(4,4)$ in $(38)$.

The last issue is to solve the problem when control specification cannot be satisfied over some frequency ranges. This means that the learning has to be performed over these frequencies where the LMI condition of Theorem 1 is satisfied. The remaining frequencies should be cut-off by a low-pass filter (which can be implemented as the zero-phase filter, e.g., by using the filtfilt routine in MATLAB) with cut-off frequency equal the highest frequency for which the result of Theorem 1 is valid.

\subsection{Uncertain dynamics}

The results of Theorems 1 and 2 can be extended to case when there is uncertainty in the model of the system to be controlled. As in other areas, it is assumed that the uncertainty lies in a particular model structure and in this paper the case considered is where the matrices $A$ and $A_{d}$ of a process (4) are not exactly known. In particular, it is assumed that dynamics are subject to timevarying structured uncertainties, resulting in dynamics to be controlled described by the state-space model

$$
\begin{aligned}
\dot{x}_{k}(t) & =(A+\delta A) x_{k}(t)+\left(A_{d}+\delta A_{d}\right) x_{k}(t-d)+B \Gamma u_{k}(t), \\
y_{k}(t) & =C x_{k}(t),
\end{aligned}
$$

where the uncertainty is assumed to be of the form

$$
\left[\begin{array}{ll}
\delta A & \delta A_{d}
\end{array}\right]=E \Delta(t)\left[\begin{array}{ll}
F & F_{d}
\end{array}\right]
$$

and $E, F, F_{d}$ are known constant matrices of compatible dimensions. Also $\Delta(t)$ is an unknown, real and possibly time-varying matrix with elements satisfying

$$
\Delta^{T}(t) \Delta(t) \leq I, \forall t \in[0, \alpha],
$$

i.e., $\Delta(t)$ belongs to a ball of matrices of unit radius $\forall t \in[0, \alpha]$.

Applying the same control law as in the ideal model case results in the controlled dynamics state-space model

$$
\begin{aligned}
\dot{\eta}_{k}(t) & =\hat{\mathcal{A}} \eta_{k}(t)+\hat{A}_{d} \eta_{k}(t-d)+\hat{\mathcal{B}}_{0} e_{k-1}(t), \\
e_{k}(t) & =\hat{\mathcal{C}} \eta_{k}(t)+\hat{C}_{d} \eta_{k}(t-d)+\hat{\mathcal{D}}_{0} e_{k-1}(t),
\end{aligned}
$$

where

$$
\begin{aligned}
& \hat{\mathcal{A}}=A+\delta A+B \Gamma K_{1}, \hat{A}_{d}=A_{d}+\delta A_{d}, \hat{\mathcal{B}}_{0}=B \Gamma K_{2}, \\
& \hat{\mathcal{C}}=-C\left(A+\delta A+B \Gamma K_{1}\right)=-C \hat{\mathcal{A}}, \hat{C}_{d}=-C\left(A_{d}+\delta A_{d}\right), \hat{\mathcal{D}}_{0}=I-C B \Gamma K_{2} .
\end{aligned}
$$

The following results are the uncertain model versions of Theorems 1 and 2 respectively. 
Theorem 3. With the notation of (34), an ILC scheme described as a differential linear repetitive process of the form (42) with uncertainty structure modelled by (40)-(41) and under the actuator fault description (5)-(8) is stable along the trial and hence monotonic trial-to-trial error convergence occurs for the performance specifications over low and middle frequency ranges (25) and any delayd satisfying $0<d \leq \bar{d}$ if there exist matrices $\hat{P} \succ 0, \hat{Q} \succ 0, \hat{X} \succ 0, \hat{Z} \succ 0, S, L$ and positive scalars $\varepsilon_{1}$ and $\lambda_{1}$ such that

$$
\left[\begin{array}{ccc}
\Theta_{11} & \lambda_{1} H_{2} & F_{2}^{T} \\
(\star) & -\lambda_{1} I & 0 \\
(\star) & (\star) & -\lambda_{1} I
\end{array}\right] \prec 0,
$$

where $\Theta_{11}$ is given by (33) and

$$
H_{2}=\left[\begin{array}{lllllll}
0 & E^{T} & 0 & 0 & -(C E)^{T} & 0 & 0
\end{array}\right]^{T}, F_{2}=\left[\begin{array}{lllllll}
0 & F S & F_{d} S & 0 & 0 & 0 & 0
\end{array}\right] .
$$

If this LMI is feasible, the control law matrices $K_{1}$ and $K_{2}$ are given by (35).

Proof. Suppose that the LMI (43) is feasible. Then application of Schur's complement formula to 43 gives

$$
\Theta_{11}+\lambda_{1} H_{2} H_{2}^{T}+\lambda_{1}^{-1} F_{2}^{T} F_{2} \prec 0,
$$

and by Lemma 1, this inequality is feasible if and only if

$$
\Theta_{11}+\operatorname{sym}\left\{H_{2} \Delta(t) F_{2}\right\} \prec 0,
$$

holds. This last inequality is (33) applied to uncertainty case and by the result of Theorem 1 feasibility of (43) ensures that a differential linear repetitive process of the form (42) is stable along the trial and the proof is complete.

Theorem 4. With the notation of (34), an ILC scheme described as a differential linear repetitive process of the form (42) with uncertainty structure modelled by (40)-(41) and under the actuator fault description (5)-(8) is stable along the trial and hence monotonic trial-to-trial error convergence occurs for performance specifications over the high frequency range (25) and any delay d satisfying $0<d \leq \bar{d}$ if there exist matrices $\hat{P} \succ 0, \hat{Q} \succ 0, \hat{X} \succ 0, \hat{Z} \succ 0, S, L$ and positive scalars $\beta, \varepsilon_{2}$ and $\lambda_{2}$ such that

$$
\left[\begin{array}{ccc}
\Theta_{12} & \lambda_{2} H_{2} & F_{2}^{T} \\
(\star) & -\lambda_{2} I & 0 \\
(\star) & (\star) & -\lambda_{2} I
\end{array}\right] \prec 0,
$$

where $\Theta_{12}$ is given in (38). If this LMI is feasible, the control law matrices $K_{1}$ and $K_{2}$ are given by (35).

Proof. This result is proved, with routine changes, in the same way as Theorem 3 . Hence the details are omitted.

For implementation, back substituting in 160$)$ for $\eta_{k}(t)$ and $e_{k}(t)$ gives

$$
u_{k}(t)=u_{k-1}(t)+K_{1}\left(x_{k}(t)-x_{k-1}(t)\right)+K_{2}\left(\dot{x}_{k}(t)-\dot{x}_{k-1}(t)\right) .
$$




\section{Case study}

To illustrate the application of the new design, a two-stage chemical reactor with delayed recycle streams is considered, where it is assumed that both of the reactors are isothermal continuous stirred tank reactors (CSTR). A reactor recycle does not increase the overall conversion and reduces the cost of a reaction and therefore it is commonly used in industrial applications. The input to be recycled must be separated from the yields and then travel through pipes. The total recycle time therefore introduces delays into the state. Consider the irreversible reaction $A_{p} \rightarrow B_{p}$ with negligible

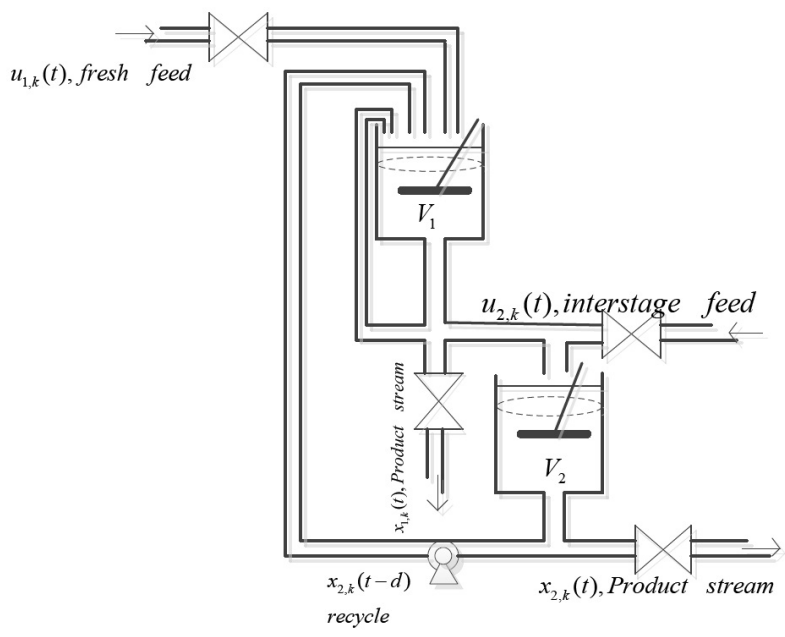

Figure 1: Two-stage chemical reactor train with delayed recycle streams.

heat effects that is completed in a two-stage reactor system. The reactor temperature is maintained constant and therefore only the composition of the product streams from the two reactors needs to be controlled. The manipulated variables are the feed compositions to the two reactors. Also, the flow rates to the reactor system are fixed and only the compositions vary.

Suppose that the fresh feed of pure $A_{p}$ is to be mixed with the recycle stream of unreacted $A_{p}$ with recycle flow rate $R$. The mass balance equations that govern the reactors shown in Fig. 11 are [24]

$$
\left\{\begin{array}{l}
\dot{x}_{1, k}(t)=-\left(\frac{1}{\theta_{1}}+k_{1}\right) x_{1, k}(t)+\frac{R}{V_{1}} x_{2, k}(t-d)+\frac{F_{1}}{V_{1}} u_{1, k}(t), \\
\dot{x}_{2, k}(t)=-\left(\frac{1}{\theta_{2}}+k_{2}\right) x_{2, k}(t)+\frac{F_{p 2}-F_{2}+R}{V_{2}} x_{1, k}(t)+\frac{F_{2}}{V_{2}} u_{2, k}(t),
\end{array}\right.
$$

where $x_{1, k}(t)$ and $x_{2, k}(t)$ are the compositions and for $i=1,2, \theta_{i}$ are the reactor residence times, $k_{i}$ are the reaction constants, $F_{1}$ is the feed rate, $F_{2}$ is the interstage feed rate and $F_{p 2}$ is the second product stream, respectively. Also $V_{i}$ are the reactor volumes and the time-delay range is $0<d \leq \bar{d}=1$, where $d=1$ is used in the simulation results given below. In operation, this time-delay differential system needs to execute the same reaction task over a finite duration and introducing

$$
x_{k}(t)=\left[\begin{array}{ll}
x_{1, k}^{T}(t) & x_{2, k}^{T}(t)
\end{array}\right]^{T}, y_{k}(t)=x_{k}(t)=\left[\begin{array}{ll}
y_{1 k}^{T}(t) & y_{2 k}^{T}(t)
\end{array}\right]^{T},
$$

the nominal system state-space model of the form (4) is obtained with $C=I$ and

$$
A=\left[\begin{array}{cc}
-\left(\frac{1}{\theta_{1}}+k_{1}\right) & 0 \\
\frac{F_{p 2}-F_{2}+R}{V_{2}} & -\left(\frac{1}{\theta_{2}}+k_{2}\right)
\end{array}\right], B=\left[\begin{array}{cc}
\frac{F_{1}}{V_{1}} & 0 \\
0 & \frac{F_{2}}{V_{2}}
\end{array}\right], A_{d}=\left[\begin{array}{cc}
0 & \frac{R}{V_{1}} \\
0 & 0
\end{array}\right], \theta_{1}=\frac{V_{1}}{F_{1}+R+F_{d}}, \theta_{2}=\frac{V_{2}}{F_{p 2}+R} .
$$


In the simulations below the model parameters used are $k_{1}=k_{2}=0.5, V_{1}=V_{2}=0.25, F_{1}=F_{2}=$ $0.25, F_{d}=0.15, F_{p 2}=0.4, R=0.1$. Suppose that the recycle flow rate $R$, the raw material feed rate $F_{1}$ and the interstage feed rate $F_{2}$ are subject to uncertainty. Then

$$
A=\left[\begin{array}{cc}
-2.5 & 0 \\
1 & -2.5
\end{array}\right], A_{d}=\left[\begin{array}{cc}
0 & 0.4 \\
0 & 0
\end{array}\right], B=\left[\begin{array}{ll}
1 & 0 \\
0 & 1
\end{array}\right], C=\left[\begin{array}{ll}
1 & 0 \\
0 & 1
\end{array}\right]
$$

and the uncertainty (see (40)) is specified by

$$
E=\left[\begin{array}{cc}
1 & 0 \\
0.5 & 1
\end{array}\right], F=\left[\begin{array}{cc}
0.4 & 0 \\
0 & 0.5
\end{array}\right], F_{d}=\left[\begin{array}{cc}
0 & 0.5 \\
0 & 0
\end{array}\right], \Delta(t)=\sin (2 t) .
$$

The state initial vector $x_{k}(0)$ and the input vector $u_{k}(0)$ are assumed to be zero $\forall k \geq 0$ and the reference trajectories, termed set-points from this point onwards, are:

$$
y_{1 d}(t)=\left\{\begin{array}{rlrl}
\frac{1}{20} t, & 0 & \leq t<40 \\
2+\frac{1}{120} t, & & 40 \leq t<100 \\
2.5+\frac{1}{300} t, & 100 & \leq t<250 \\
3 & 250 & \leq t \leq 300
\end{array}\right.
$$

$$
y_{2 d}(t)=\left\{\begin{array}{cc}
\frac{1}{120} t, & 0 \leq t<60 \\
0.5+\frac{1}{60} t, & 60 \leq t<120 \\
1.5, & 120 \leq t<150 \\
1.5+\frac{1}{100} t, & 150 \leq t<250 \\
2.5, & 250 \leq t \leq 300
\end{array}\right.
$$

Applying the Fast Fourier Transform (FFT) (sampling at $300 \mathrm{~Hz}$ ) gives the plot of Figure 2 where the significant harmonics are in the range 0 to $10 \mathrm{~Hz}$, which is taken as the low frequency range. Hence $\varpi_{l}=62.84 \mathrm{rad} / \mathrm{sec}$.

Partial degradation and wear from repeated control operation could lead to faults arising during the trials and it is assumed that an actuator fault in the operation valve for the feed rate could occur in this low frequency range. As a numerical example, it is assumed that $0.5 \leq \Gamma \leq \Gamma \leq \bar{\Gamma}=1$ and hence $q=\operatorname{diag}\{0.75,0.75\}, q_{0}=\operatorname{diag}\{0.33,0.33\}$. It is also assumed that no actuator faults occur arise before $k=25$ trials have elapsed.

To evaluate tracking performance from trial-to-trial, let $e_{i k}, i=1,2$ denote the errors on trial $k$. Then the convergence measure is the root mean square (RMS):

$$
\operatorname{RMS}(i k)=\frac{1}{300} \int_{0}^{300}\left(y_{i d}(t)-y_{i k}(t)\right)^{2} \mathrm{~d} t
$$

where the smaller the value of this quantity the better the tracking performance along the $k$ th trial. Next two possible scenarios are considered.

\section{Scenario 1. Nominal system with a constant fault}

Consider the case when the operating valve is always partially blocked in the reaction process (47). This constant partial fault in the nominal system causes the actuator to drop to $70 \%$ of its normal value from 20 th trial onwards. Hence $\Gamma=\operatorname{diag}\{0.7,0.7\}$ and completing the ILC design gives the corresponding matrices in $(35)$ as

$$
K_{1}=\left[\begin{array}{cc}
1.6914 & -0.0301 \\
-0.5838 & 1.6821
\end{array}\right], K_{2}=\left[\begin{array}{cc}
0.5513 & 0 \\
0 & 0.5513
\end{array}\right],
$$



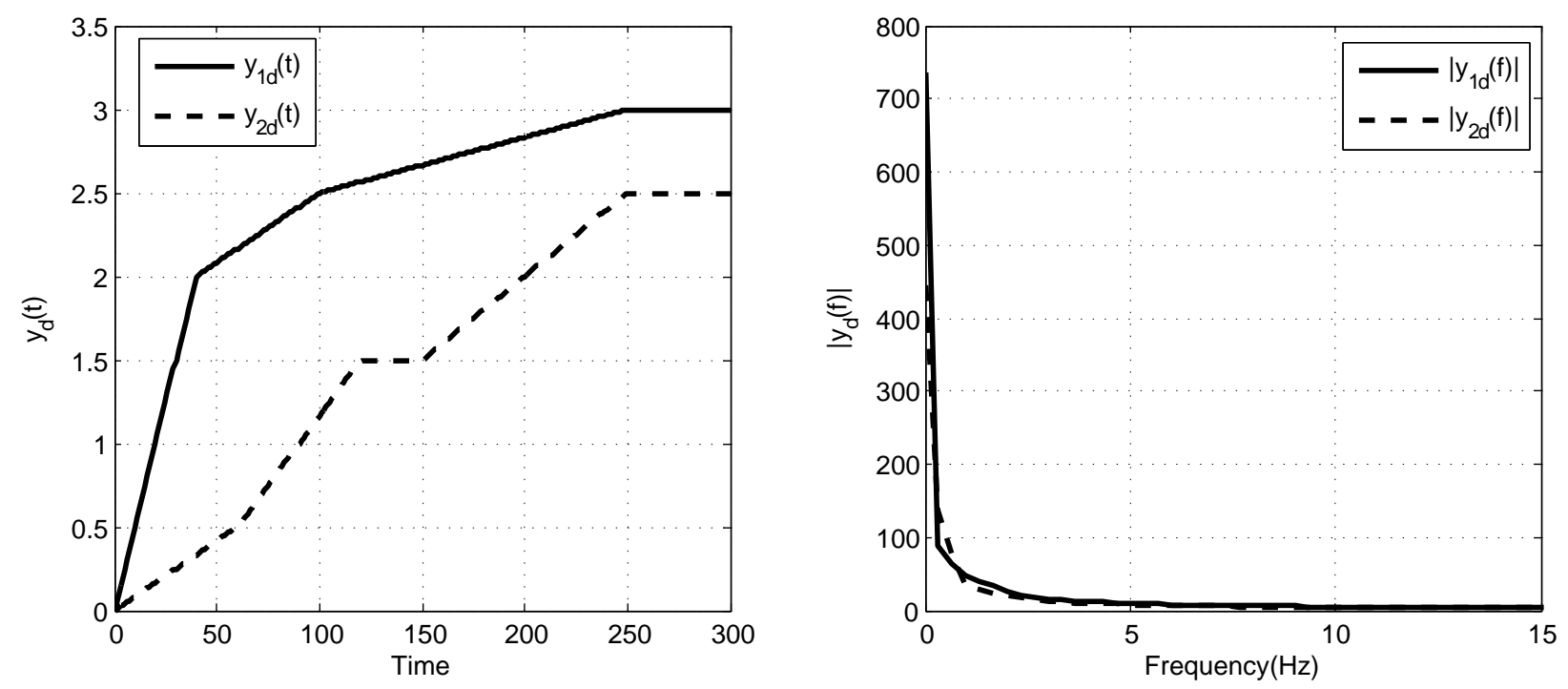

Figure 2: The output reference trajectory (a) and its corresponding frequency spectrum (b).

and the achieved minimum performance level $\mu$ in the chosen frequency range is 0.4762 . The simulation results obtained for this design are shown in Figs. 3 7. B. Before the fault occurs, the tracking errors display the monotonic decreasing property with rapid reduction from trial-to-trial. Once the fault on trial 25 occurs, the tracking performance deteriorates for some subsequent trials but then recovers to achieve close tracking again.

\section{Scenario 2. Uncertain system with a time-varying fault}

In this scenario, the operation valve is partly blocked by a time-varying fault in the reaction process (47). In this case the time-varying fault matrix $\Gamma=\operatorname{diag}\{0.6+0.1 \sin (t), 0.6+0.1 \sin (t)\}$ is used from trial 25. Solving the LMI of (43) in Theorem 3 gives the control law matrices

$$
K_{1}=\left[\begin{array}{cc}
1.7673 & -0.0321 \\
-0.8766 & 1.8847
\end{array}\right], K_{2}=\left[\begin{array}{cc}
0.5702 & 0 \\
0 & 0.5702
\end{array}\right]
$$

and the achieved minimum performance level $\mu$ in the chosen frequency range is 0.5020 . The simulation results obtained for this design are shown in Figs. 8 8 12. These confirm that the fault does affect the outputs produced and hence the tracking performance but this is still a baseline acceptable design.

\section{Conclusion}

This paper has considered the iterative learning fault tolerant tracking control problem for a class of differential linear time-delay batch processes. Based on the repetitive process setting and the generalized KYP lemma, a fault-tolerant control law design has been developed and extended to the uncertain model case. The KYP lemma setting enables the application relative case of imposing performance specified over finite frequency ranges to be included. To highlight the new results, a simulation-based study on the model of a two-stage chemical reactor with delayed recycle streams has been given.

These results are the first on this approach and there are many areas to which further research could profitably be directed. One area is that the current control law is state feedback based and if all states are not available for measurement either an observer is required or the theory is extended 

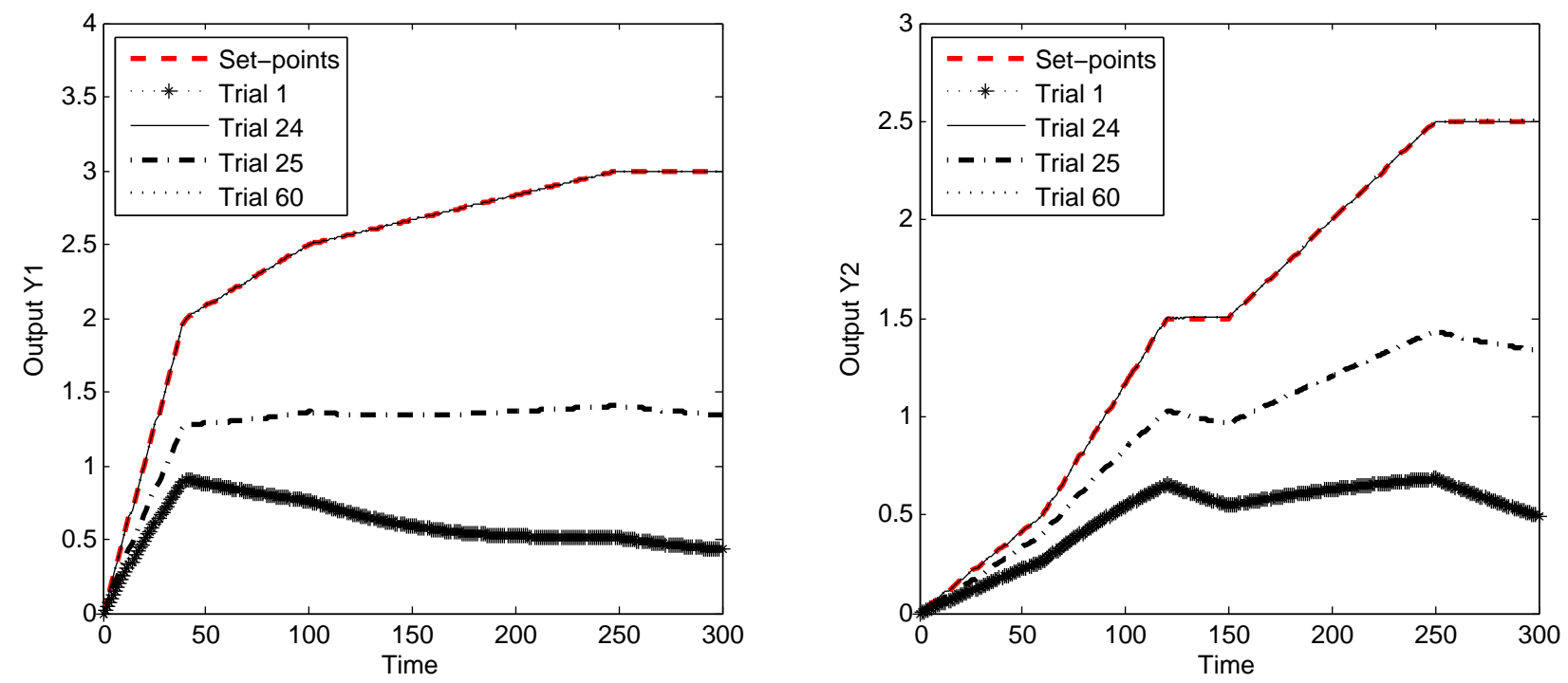

Figure 3: The set-points and tracking performance for the controlled dynamics with and without faults for scenario 1.

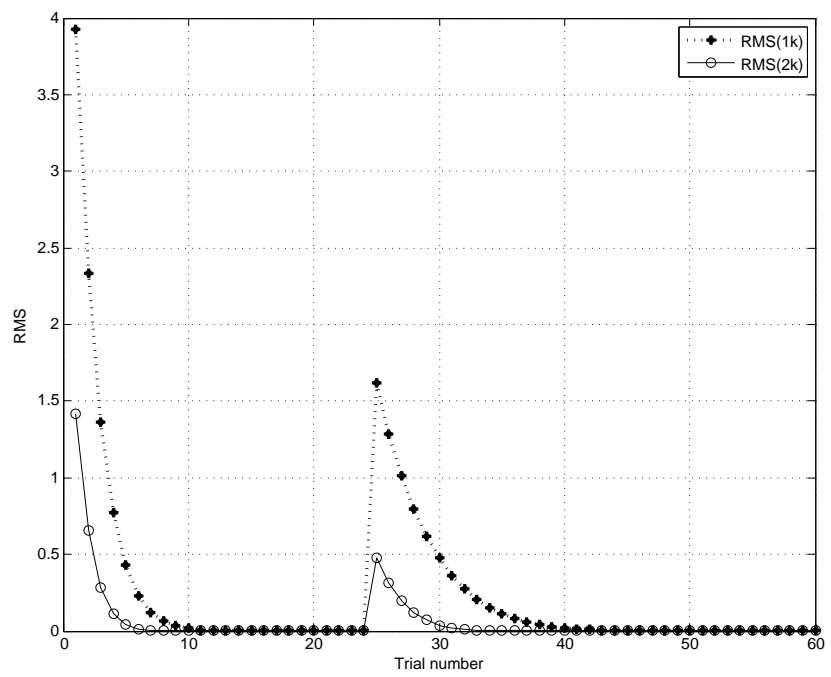

Figure 4: The RMS performance against trial number for scenario 1. 

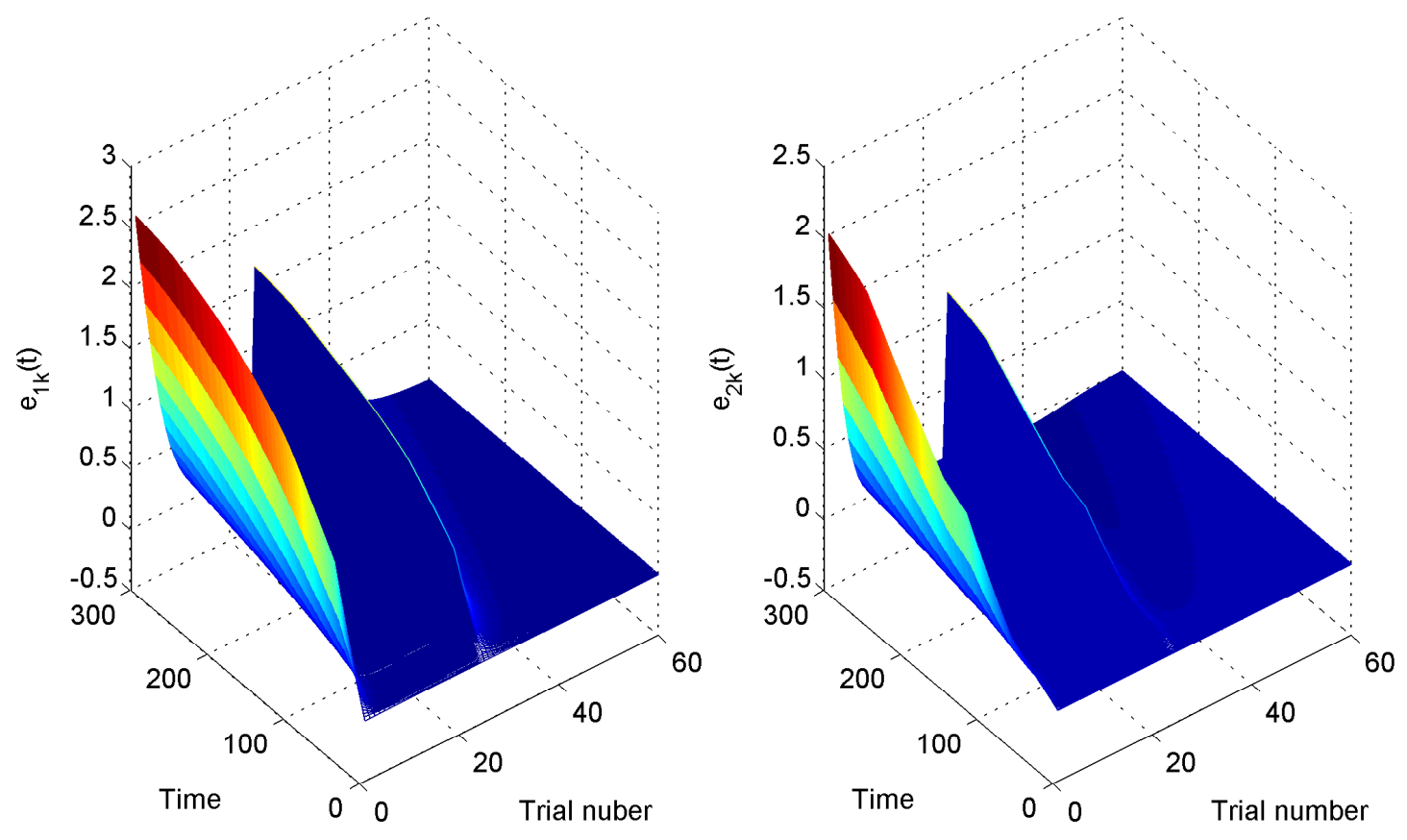

Figure 5: The tracking errors for scenario 1.
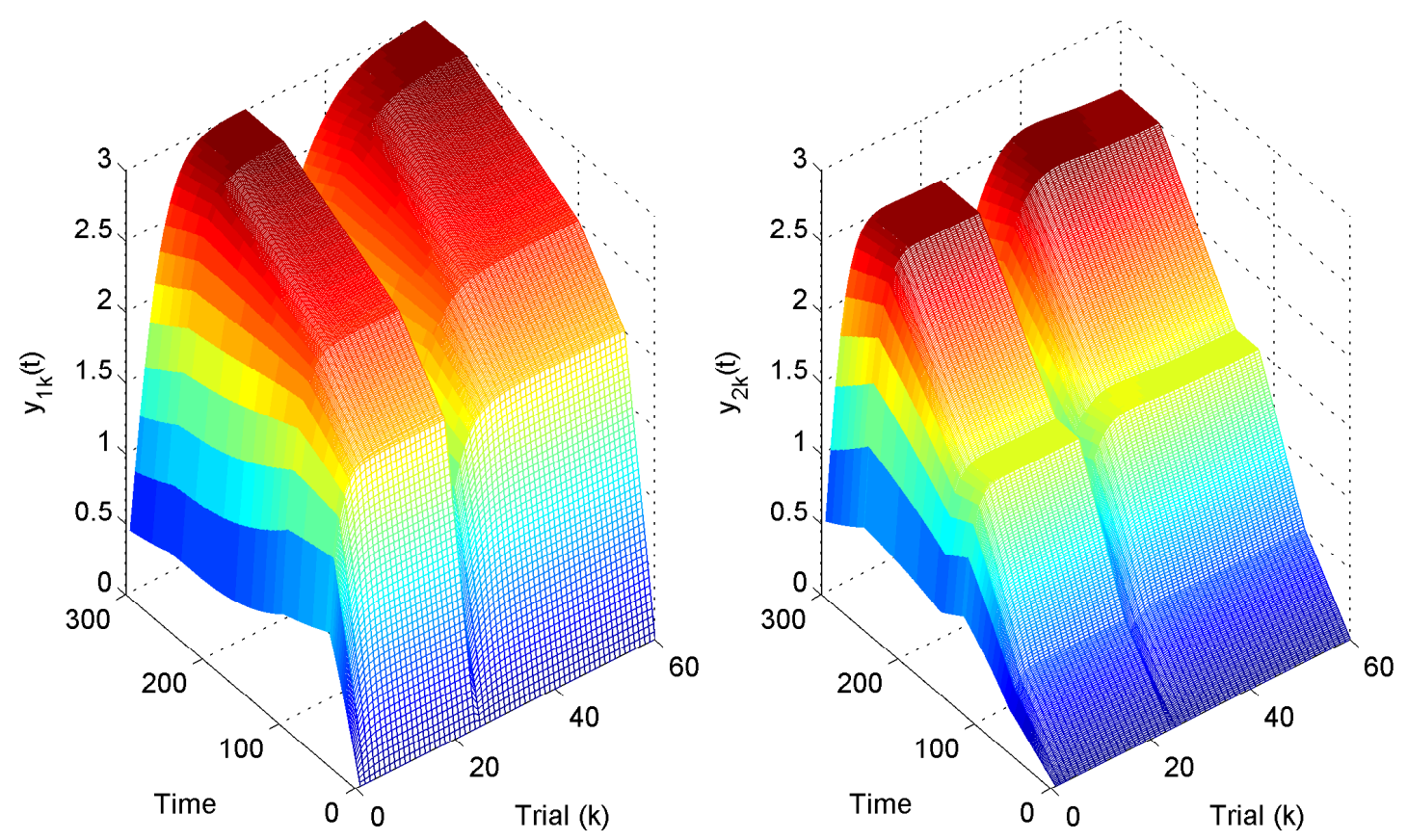

Figure 6: The outputs for scenario 1. 

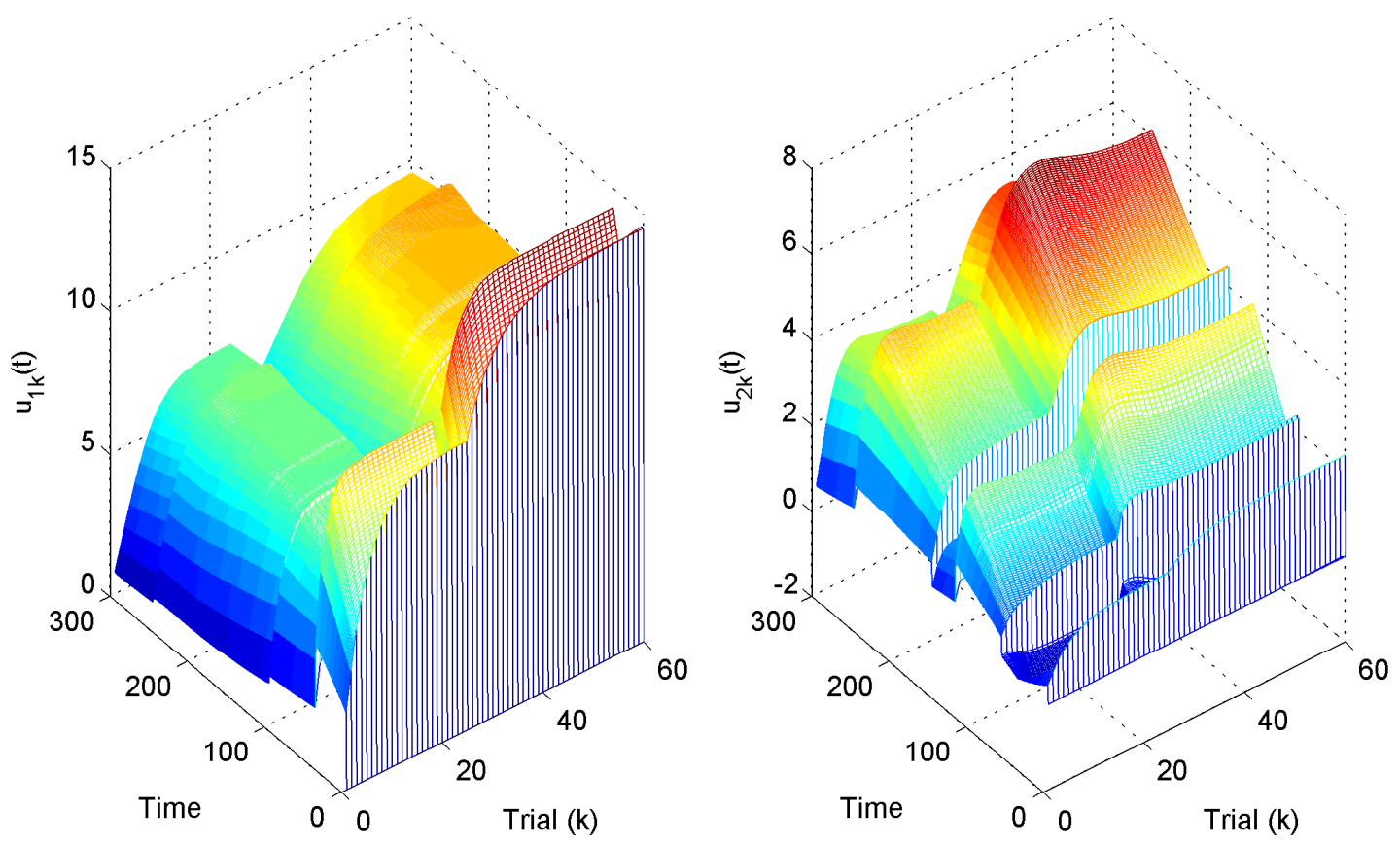

Figure 7: The control inputs for scenario 1.
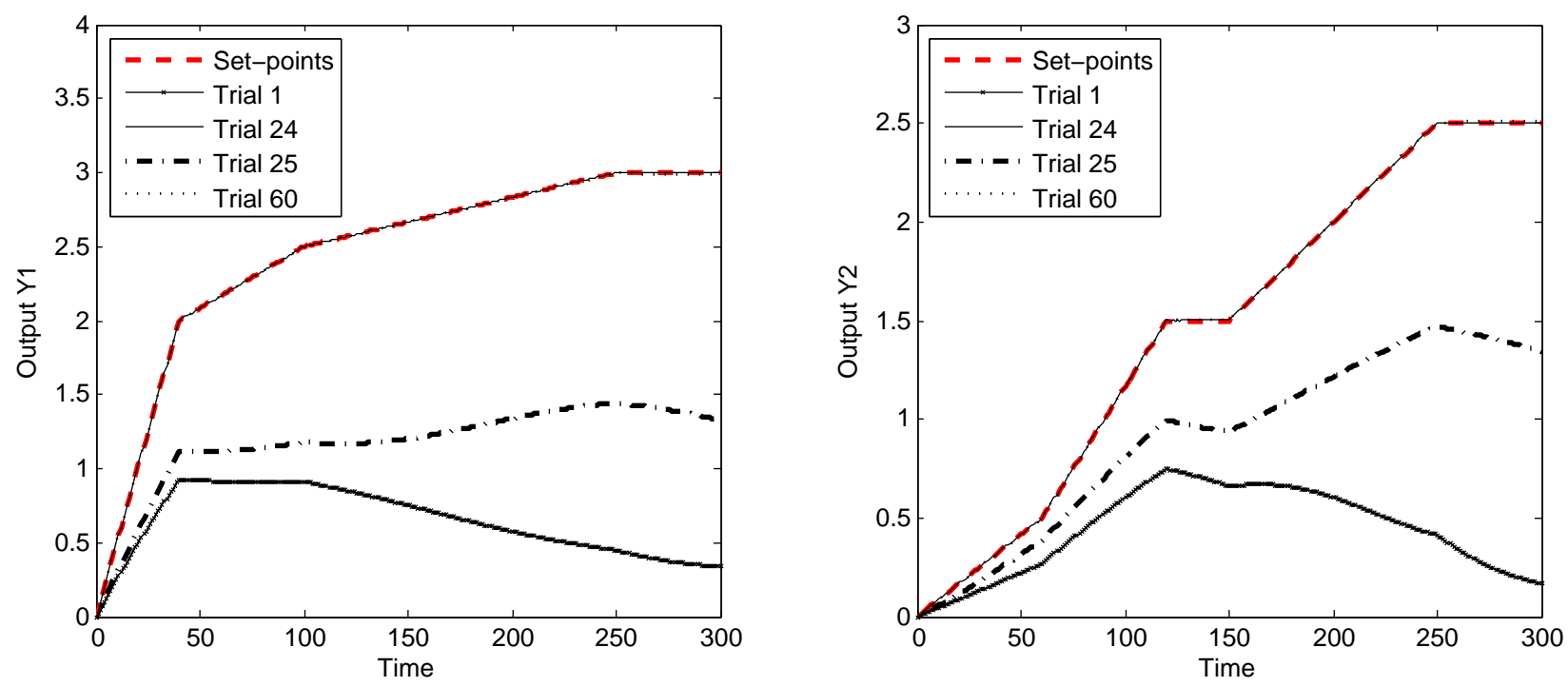

Figure 8: The outputs for scenario 2. 


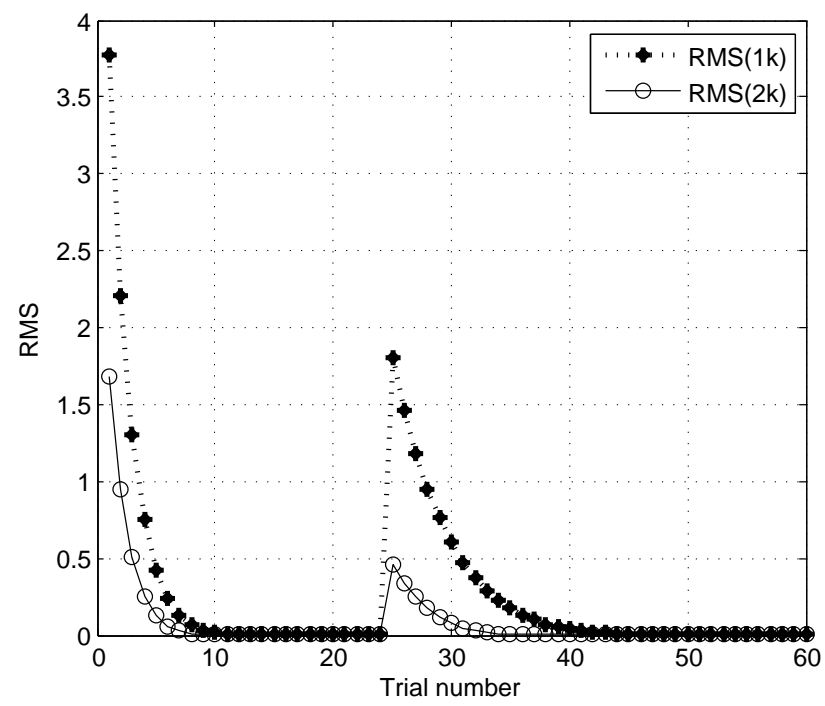

Figure 9: The RMS performance against trial number for scenario 2.
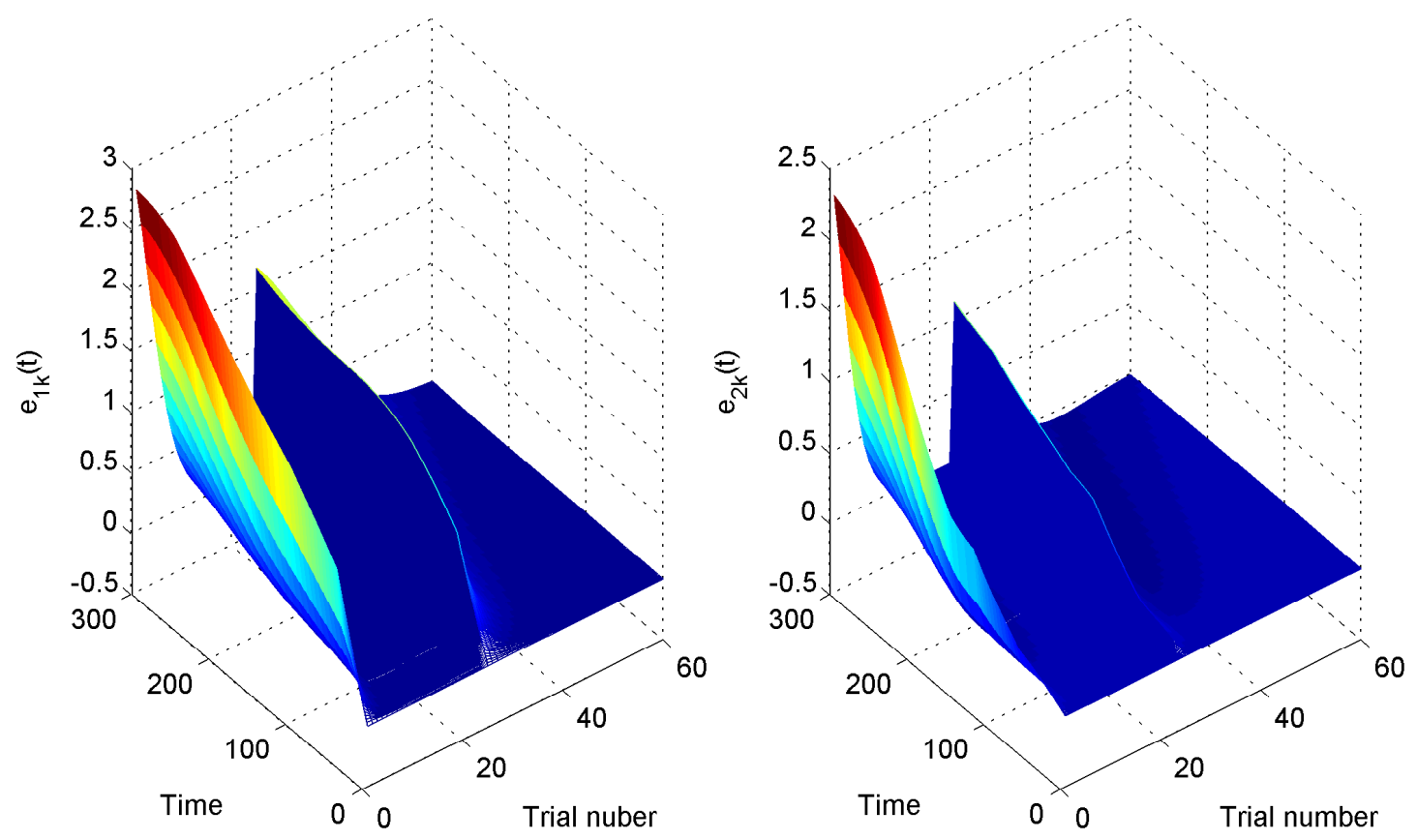

Figure 10: The tracking errors for scenario 2. 

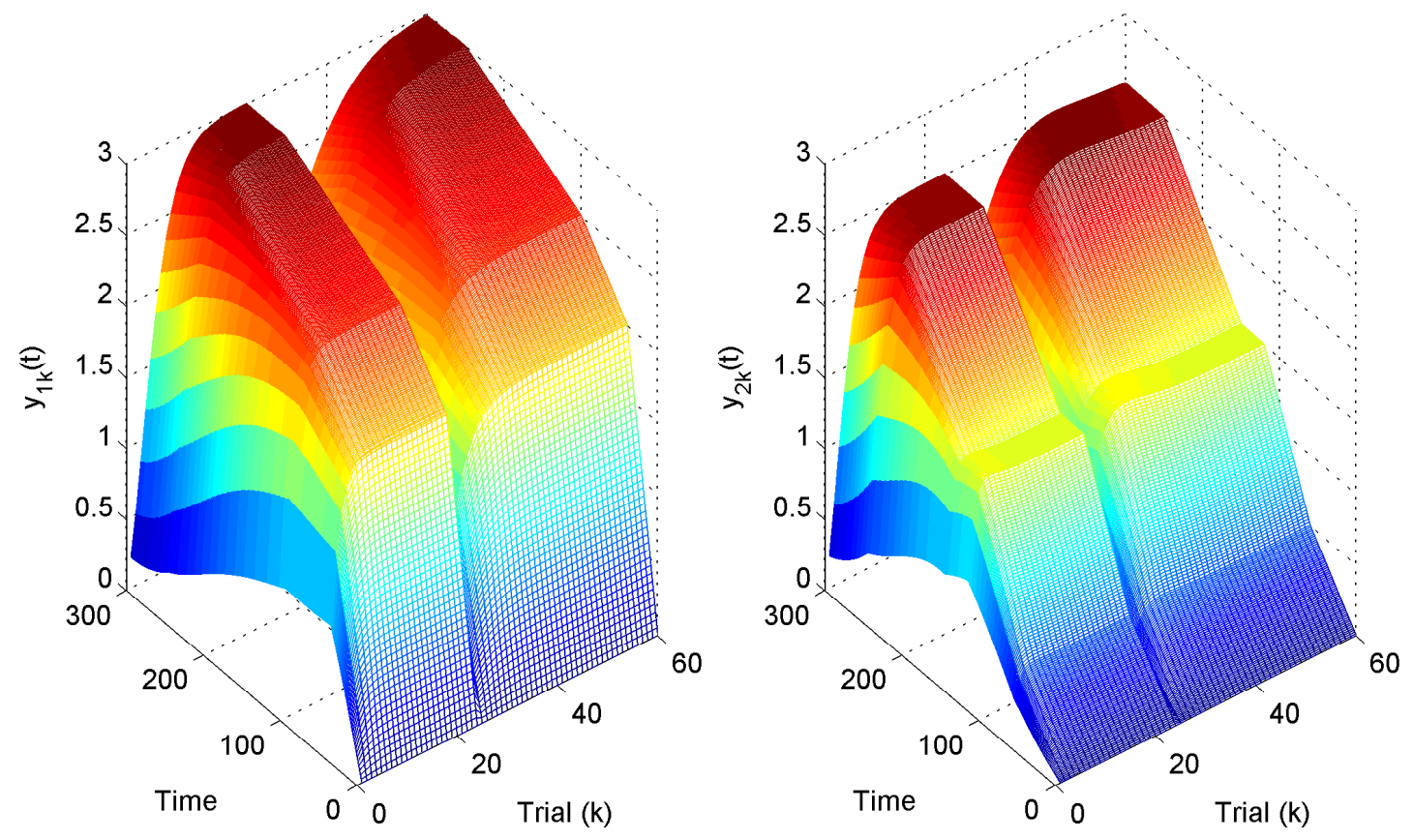

Figure 11: The outputs for scenario 2.
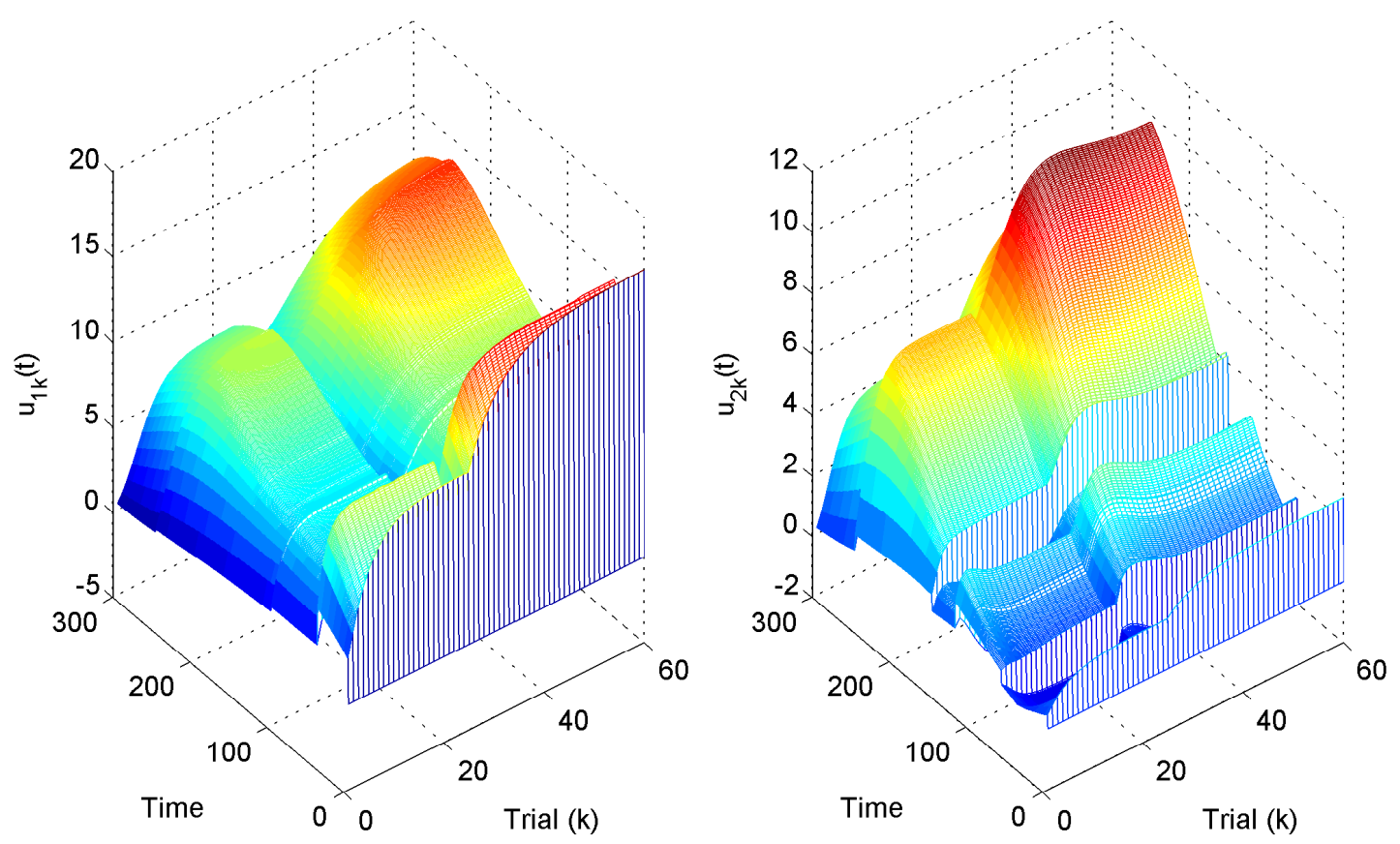

Figure 12: The control inputs for scenario 2. 
to a control law that uses only output information. Given that the trial length is finite and all data is available once a trial is complete, zero-phase filtering can be applied to the previous trial data before computing the next control mitigate against unmeasurable disturbance effects. Extending the theory to include disturbance attenuation is also a possible topic for future work. Also the inclusion of $\mathcal{H}_{\infty} / \mathcal{H}_{2}$ performance measures is another area for possible future research.

\section{References}

[1] Arimoto, S., Kawamura, S., and Miyazaki, F. (1984), "Bettering operation of robots by learning," Journal of Robotic Systems, vol. 1, no 2, pp. 123-140.

[2] Ahn, H. S., Chen, Y. Q., and Moore, K. L. (2007), "Iterative learning control: brief survey and categorization," IEEE Transactions on Systems, Man and Cybernetics, Part C: Applications and Reviews, vol. 37, no 6, pp. 1109-1121.

[3] Bristow, D. A., Tharayil, M., and Alleyne, A. (2006), "A survey of Iterative Learning Control," IEEE Control Systems Magazine, vol. 26, no 3, pp. 96-114.

[4] Hładowski, Ł., Gałkowski, K., Cai, Z., Rogers, E., Freeman, C. T. and Lewin, P. L. (2010), "Experimentally supported 2D systems based iterative learning control law design for error convergence and performance," Control Engineering Practice, vol. 18, no. 4, pp. 339-348.

[5] Barton, K. L., and Alleyne, A. G. (2011), "A norm optimal approach to time-varying ILC with application to a multi-axis robotic testbed," IEEE Transactions on Control Systems Technology, vol. 19 , no. 1 , pp. $166-180$.

[6] Bolder, J., and Oomen, T. (2015), "Rational basis functions in iterative learning control - with experimental verification on a motion system," IEEE Transactions on Control Systems Technology, vol. 23, no. 2, pp. 722-729.

[7] Heinzen, A., Gillella, P., Sun, Z., (2011), "Iterative learning control of a fully flexible valve actuation system for non-throttled engine load control," Control Engineering Practice, vol. 19, no 12 , pp. $1490-1505$.

[8] Jia, L., Yang, T., and Chiu, M. S. (2013), "An integrated iterative learning control strategy with model identification and dynamic R-parameter for batch processes," Journal of Process Control, vol. 23, no 9, pp. 1332-1341.

[9] Wang, Y., Gao, F. and Doyle, F. J. (2009), "Survey on iterative learning control, repetitive control, and run-to-run control," Journal of Process Control, vol. 19, no. 10, pp. 1589-1600.

[10] Freeman, C. T., Hughes, A.-M., Burridge, J. H., Chappell, P. H., Lewin, P. L., and Rogers, E., (2009), "Iterative learning control of FES applied to the upper extremity for rehabilitation." Control Engineering Practice, vol. 17, pp. 368-381.

[11] Meadmore, K. L., Exell, T., Hallewell, E., Hughes, A-M., Freeman, C. T., Kutlu, M., Benson, V., Rogers, E., and Burridge, J. H. (2014), "The application of precisely controlled functional electrical stimulation to the shoulder, elbow and wrist for upper limb stroke rehabilitation: a feasibility study," Journal of NeuroEngineering and Rehabilitation, vol. 11, no 105.

[12] Kurek, J., and Zaremba, M. (1993), "Iterative learning control synthesis based on 2-D System Theory," IEEE Transactions on Automatic Control, vol. 38, no 1, pp. 121-125. 
[13] Rogers, E., Gałkowski, K., and Owens, D. H., Control Systems Theory and Applications for Linear Repetitive Processes, vol. 349 of Lecture Notes in Control and Information Sciences, Berlin, Germany: Springer-Verlag (2007).

[14] Liu, T., and Gao, F. R. (2010), "Robust two-dimensional iterative learning control for batch processes with stae delay and time-varying uncertainties," Chemical Engineering Science, vol. 65, no. 23, pp. 6134-6144.

[15] Kim, K. K., Skogestad, S., and Morari, M. (2014), "Necessary and sufficient conditions for robust reliable control in the presence of model uncertainties and system component failures," Computers and Chemical Engineering, vol. 70, no 11, pp. 67-77.

[16] Paszke, W., Rogers, E. and Gałkowski, K. (2016), "Experimentally verified generalized KYP lemma based iterative learning control design," Control Engineering Practice, vol. 53, pp. 5767.

[17] Paszke, W., Rogers, E. and Gałkowski, K. (2013), "KYP lemma based stability and control law design for differential linear repetitive processes with applications," Systems and Control Letters, vol. 67, no 7, pp. 560-566.

[18] Xie, L. H. (1996), "Output feedback $\mathcal{H}_{\infty}$ control of systems with parameter uncertainty," International Journal of Control, vol. 63, no 4, pp. 741-750.

[19] Yu, L. (2005), "An LMI approach to reliable guaranteed cost control of discrete-time systems with actuator failure," Applied Mathematics and Computation, vol. 162, no 3, pp. 1325-1331.

[20] Gahinet, P., and Apkarian, P. (1994), "A linear matrix inequality approach to $\mathcal{H}_{\infty}$ control," International Journal of Robust and Nonlinear Control, vol. 4, no 4, pp. 421-448.

[21] Pipeleers, G., and Vandenberghe, L. (2011), "Generalized KYP lemma with real data," IEEE Transactions on Automatic Control, vol. 56, no 12, pp. 2942-2946.

[22] Longman, R. W. (2000), "Iterative learning and repetitive control for engineering practice," International Journal of Control, vol. 73, no. 930-954.

[23] Zhang, X. N., and Yang, G. H. (2012), "Performance analysis for multi-delay systems in finite frequency domains.," International Journal of Robust and Nonlinear Control, vol. 22, no 8, pp. 933-944.

[24] Nguang, S. K. (2000), "Robust stabilization of a class of time-delay nonlinear systems," IEEE Transactions on Automatic Control, vol. 45, no 4, pp. 756-762. 